\title{
Gyrokinetic Theory for Arbitrary Wavelength Electromagnetic Modes in Tokamaks
}

\author{
H. Qin, W. M. Tang, and G. Rewoldt \\ Princeton Plasma Physics Laboratory \\ Princeton University, Princeton, NJ 08543
}

October 15,1997

\begin{abstract}
A linear gyrokinetic system for arbitrary wavelength electromagnetic modes is developed. A wide range of modes in inhomogeneous plasmas, such as the internal kink modes, the toroidal Alfvén eigenmode (TAE) modes, and the drift modes, can be recovered from this system. The inclusion of most of the interesting physical factors into a single framework enables us to look at many familiar modes simultaneously and thus to study the modifications of and the interactions between them in a systematic way. Especially, we are able to investigate selfconsistently the kinetic MHD phenomena entirely from the kinetic side. Phase space Lagrangian Lie perturbation methods and a newly developed computer algebra package for vector analysis in general coordinate system are utilized in the analytical derivation. In tokamak geometries, a $2 \mathrm{D}$ finite element code has been developed and tested. In this paper, we present the basic theoretical formalism and some of the preliminary results.
\end{abstract}


$52.25 \mathrm{Fa}, 52.25 \mathrm{Dg}, 52.35 \mathrm{Py}$

\section{Introduction}

The motivation of this research project is twofold: to develop an alternative, more comprehensive and self-consistent approach for kinetic MHD theory, and to add electromagnetic effects to a global kinetic analysis of low frequency microinstabilities with the goal of achieving a better understanding of anomalous transport in toroidal geometry. Basically, the previous focus has been on the electrostatic drift type instabilities and on pure fluid type MHD modes. However, in order to realistically assess the stability properties in high temperature (high beta) plasmas, it becomes necessary to systematically analyze kinetic MHD modes and electromagnetic drift waves. Developing the required methods of analysis and the associated codes constitute fundamental problems in the field of plasma stability. It is believed that the interaction between kinetic effects and MHD modes, such as the fishbone modes and the TAE modes, is the key physical reason for many bewildering phenomena in fusion plasmas. In addition, there are possible new applications of kinetic-MHD, such as collisionless reconnection, which is thought to be relevant to magnetic storms in the magnetosphere and to the sawtooth instability commonly seen in modern tokamaks. For drift type microinstabilities and the associated transport theory, the inclusion of electromagnetic effects has long been recognized as being necessary. For example, the examination of electromagnetic $\eta_{i}$ modes in slab geometry [1] and in toroidal geometry [2,3] revealed that increasing plasma beta can provide a stabilizing effect, especially when finite Larmor radius (FLR) effects of ions become important.

These problems can be put into a single theoretical framework - the gyrokinetic the- 


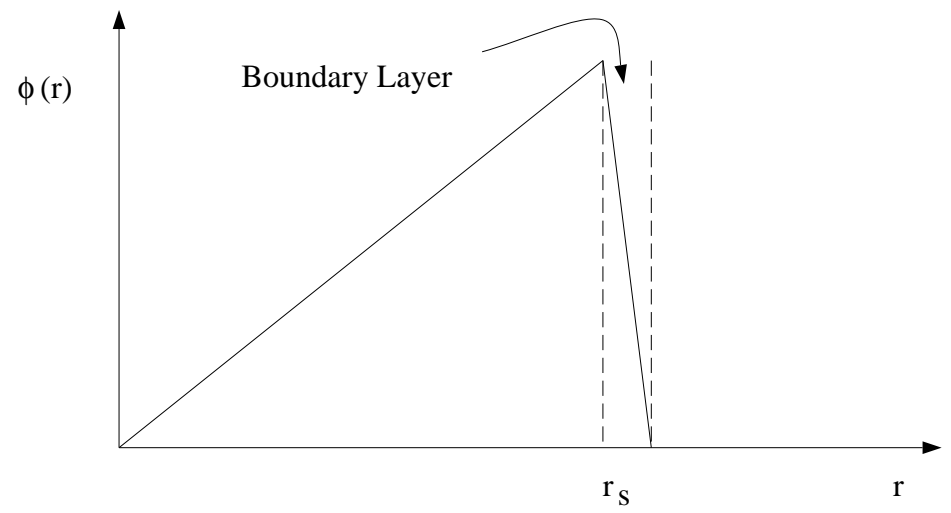

Figure 1: The multi-scale-length structure of the internal kink mode.

ory of arbitrary wavelength electromagnetic modes. On the one hand, including magnetic components in the kinetic analysis and extending it to long wavelength modes formally leads us into the kinetic-MHD regime from the kinetic side. An example of this approach is the kinetic MHD ballooning mode theory. [4] Using this formalism we are able to recover those familiar MHD results entirely from the kinetic point of view, and more importantly to obtain kinetic modifications. Compared with previous hybrid kinetic-MHD theory, the approach from the kinetic side is more rigorous, selfconsistent and comprehensive. On the other hand, the drift type microinstabilities and the associated transport can be also investigated systematically in this theoretical framework. Not only do we recover the existing results such as the electrostatic limit, [5, 6] the long wavelength limit, [7] and the ballooning limit, but also we can explore many new problems, for example, the intermediate wavelength regime and the coupling between drift waves and shear Alfven waves.

Furthermore, in magnetized plasmas there exist a lot of multi-scale-length modes. Actually the well-known internal kink mode is indeed a multi-scale-length mode (See Figure 1).

For an unstable internal kink mode, there is a boundary layer around the rational surface, 
inside which the scale length is much shorter than that outside. FLR effects are important inside the boundary layer, whereas outside the boundary layer it is just a long wavelength MHD mode. Obviously this structure can not be described by the conventional approaches, neither the long wavelength ideal MHD nor the short wavelength kinetic theory. An arbitrary wavelength kinetic approach will provide us with a tool for this kind of multi-scale-length structure.

In this paper, we present our gyrokinetic theory for arbitrary wavelength electromagnetic modes. First, the linear gyro-kinetic equation (GKE) valid for arbitrary wavelength is derived using the phase space Lagrangian Lie perturbation method. [8, 9, 10, 11, 12] The existing gyrokinetic equations are mainly derived for the high modenumber (i.e. short wavelength) modes, [19] for which some of the background inhomogeneities are not important and are left out. However the most crucial physical factors driving the long wavelength modes, such as the toroidal Alfvén eigenmode (TAE)[13, 14] mode and the internal kink mode,[15, 16, 17] are the background inhomogeneities which include the inhomogeneities of the magnetic field, temperature and density. Part of the inhomogeneity of the magnetic field enters through the current distribution. In our GKE, all the background inhomogeneities are fully retained. Then a gyrokinetic system for the shear Alfvén modes is developed. This system consists three basics equations: the gyrokinetic equation, the gyrokinetic quasineutrality condition, and the gyro-kinetic moment equation (GKM) which is derived by combining the parallel Ampere's law and the 0th moment of the GKE. In this system, all the interesting physical factors are kept. Many classical results obtained before by different theories can be put into a single framework in our theory. Therefore, it is also a good framework to study the modifications of and the interactions between these classical modes.

The solution methods for this system are also developed. Even though this is a fully 
kinetic approach, the differential equations which need to be solved numerically can be cast rigorously into the configuration dimensions. This is accomplished by solving the linear GKE using the method of integrating along characteristic lines, and substituting the solution of the distribution function in terms of perturbed fields back into the quasineutrality condition and the GKM. A 2D numerical code for tokamak geometries has been developed and tested. $[6,7]$ The newly developed computer algebra package for vector analysis in general coordinate systems is also utilized in solving the equation system.[18] In section 2, we derive the linear GKE for arbitrary wavelength modes and the corresponding gyrokinetic Maxwell's equations. The gyrokinetic system for shear Alfvén physics is presented in section 3. Then, in section 4 we compare our system to other existing equations and especially the ideal MHD equation. Section 5 is about the analytical and numerical solution methods. Two simple applications, the local dispersion relations for electrostatic drift waves and the instabilities of the internal kink mode in a straight tokamak, are given in section 6 . The last section is the conclusions and some discussion on our future work.

\section{Linear gyrokinetic equation for arbitrary wavelength electromagnetic modes}

Different versions of the GKE have been derived many times by different methods in different representations.[19, 20, 21, 22, 11, 12] Usually, it is derived for short wavelength modes for which many of the equilibrium inhomogeneities can be neglected. However the essence of the GKE is to average out the fast time scale gyromotion. The wavelength can be left unspecified and all the equilibrium inhomogeneities can be kept in. We will derive the linear GKE for arbitrary wavelength modes using the phase space Lagrangian Lie perturbation method. 
The derivation here is similar to that of Brizard for the nonlinear GKE.[12] However, here we consider arbitrary wavelength modes, and all the equilibrium inhomogeneities are fully retained. We use the $U$ representation instead of the $\rho_{\|}$representation, where $U$ is the parallel velocity and $\rho_{\|} \equiv U / \Omega$. Also, unnormalized real physical units are used.

The equilibrium is assumed to be magnetostatic. In the extended guiding center coordinates $(\boldsymbol{X}, U, \mu, \xi, w, t)$, the extended phase space Lagrangian is $[8,10,12]$

$$
\begin{aligned}
\gamma_{E} & =\widehat{\gamma}_{E}-H_{E} d \tau \\
& =\left(\frac{e}{c} \boldsymbol{A}+m U \boldsymbol{b}-\mu \frac{m c}{e} \boldsymbol{W}\right) \cdot d \boldsymbol{X}+\frac{m c}{e} \mu d \xi-w d t-(H-w) d \tau
\end{aligned}
$$

where $\boldsymbol{X}$ is the configuration component of the guiding center coordinate, $U$ is the parallel velocity, $\mu$ is the magnetic moment, $\xi$ is the gyrophase angle, and

$$
\boldsymbol{W}=\boldsymbol{R}+\frac{\boldsymbol{b}}{2}(\boldsymbol{b} \cdot \nabla \times \boldsymbol{b}), \quad \boldsymbol{R}=\left(\nabla \boldsymbol{e}_{1}\right) \cdot \boldsymbol{e}_{2}
$$

$\boldsymbol{b}=\boldsymbol{B} / B . \boldsymbol{e}_{1}$ and $\boldsymbol{e}_{2}$ are unit vectors in two arbitrarily chosen perpendicular directions, and $\boldsymbol{e}_{1}$ and $\boldsymbol{e}_{2}$ are perpendicular to each other. To deal with the time-dependent Hamiltonian, the regular phase space is extended to include the time coordinate and its conjugate coordinate energy $w . \widehat{\gamma}_{E}$ is the extended symplectic structure, $H_{E}=H-w$ is the extended Hamiltonian, and $H$ is the regular Hamiltonian defined as

$$
H=\frac{m U^{2}}{2}+\mu B
$$


The corresponding Poisson bracket is obtained by inverting the symplectic structure $\widehat{\gamma}_{E i j}$,

$$
\begin{aligned}
\{F, G\}= & \frac{e}{m c}\left(\frac{\partial F}{\partial \xi} \frac{\partial G}{\partial \mu}-\frac{\partial F}{\partial \mu} \frac{\partial G}{\partial \xi}\right)-\frac{c \boldsymbol{b}}{e B_{\|}^{*}} \cdot\left[\left(\nabla F+\boldsymbol{W} \frac{\partial F}{\partial \xi}\right) \times\left(\nabla G+\boldsymbol{W} \frac{\partial G}{\partial \xi}\right)\right] \\
& +\frac{B^{*}}{m B_{\|}^{*}} \cdot\left[\left(\nabla F+\boldsymbol{W} \frac{\partial F}{\partial \xi}\right) \frac{\partial G}{\partial U}-\left(\nabla G+\boldsymbol{W} \frac{\partial G}{\partial \xi}\right) \frac{\partial F}{\partial U}\right]+\left(\frac{\partial F}{\partial w} \frac{\partial G}{\partial t}-\frac{\partial F}{\partial t} \frac{\partial G}{\partial w}\right)
\end{aligned}
$$

where

$$
\boldsymbol{B}^{*}=\boldsymbol{B}+U \nabla \times \boldsymbol{b}, \quad B_{\|}^{*}=\boldsymbol{b} \times \boldsymbol{B}^{*}
$$

When the perturbed electromagnetic field is introduced, the extended phase space Lagrangian is perturbed accordingly:

$$
\begin{aligned}
\gamma_{E} & =\gamma_{E 0}+\gamma_{E 1}, \\
\gamma_{E 1} & =\left[\frac{e}{c} \boldsymbol{A}_{1}\left(T_{G C *}^{-1} \boldsymbol{X}, t\right) \cdot d\left(T_{G C *}^{-1} \boldsymbol{X}\right)\right]-e \phi_{1}\left(T_{G C *}^{-1} \boldsymbol{X}, t\right) d \tau,
\end{aligned}
$$

where $T_{G C *}$ is the push-forward transformation induced by the guiding center transformation, and $T_{G C *}^{-1}$ is its inverse.

$$
T_{G C *}^{-1} \boldsymbol{X}=\boldsymbol{X}+\boldsymbol{\rho}_{0}+\boldsymbol{\rho}_{1}+O\left(\epsilon_{B}^{2}\right)
$$

where

$$
\boldsymbol{\rho}_{0} \equiv \frac{c}{e} \sqrt{\frac{2 \mu}{B}} \widehat{\boldsymbol{\xi}},
$$

and $\epsilon_{B}$ is the ratio between the gyroradius and the scale length of the equilibrium magnetic 
field.

$$
\epsilon_{B}=\frac{\rho_{0}}{L_{B}}
$$

To derive the linear GKE, we usually don't need higher orders of the guiding center transformation. The leading order expression,

$$
T_{G C *}^{-1} \boldsymbol{X}=\boldsymbol{X}+\boldsymbol{\rho}_{0}
$$

will be sufficient for our purpose. Expanding $d\left(T_{G C *}^{-1} \boldsymbol{X}\right)$, we obtain:

$$
\gamma_{E 1}=\frac{e}{c} \boldsymbol{A}_{1}\left(\boldsymbol{X}+\boldsymbol{\rho}_{0}, t\right) \cdot\left[\left(1+\nabla \boldsymbol{\rho}_{0}\right) \cdot d \boldsymbol{X}+\frac{\partial \boldsymbol{\rho}_{0}}{\partial \mu} d \mu+\frac{\partial \boldsymbol{\rho}_{0}}{\partial \xi} d \xi\right]-e \phi_{1}\left(\boldsymbol{X}+\boldsymbol{\rho}_{0}, t\right) d \tau
$$

The essence of the Lie perturbation method is to introduce a near identity transformation from the equilibrium guiding center coordinates $Z=(\boldsymbol{X}, U, \mu, \xi, w, t)$ to the gyrocenter coordinates $\overline{\boldsymbol{Z}}=(\overline{\boldsymbol{X}}, \bar{U}, \bar{\mu}, \bar{\xi}, \bar{w}, \bar{t})$ when the perturbed field is present such that the transformed extended phase space Lagrangian $\bar{\gamma}$ can be gyrophase independent.

We emphasize that there are three different coordinate systems appearing in our formalism. $(\boldsymbol{X}, \boldsymbol{V})$ is the particle 'physical' coordinate system. $\boldsymbol{Z}=(\boldsymbol{X}, U, \mu, \xi, w, t)$ is the (extended) 'guiding center' coordinate system in an equilibrium magnetic field. When the time-dependent electromagnetic field is introduced, we use the 'gyrocenter' coordinate system $\overline{\boldsymbol{Z}}=(\overline{\boldsymbol{X}}, \bar{U}, \bar{\mu}, \bar{\xi}, \bar{w}, \bar{t})$ to describe the gyrocenter motion. Among other things, the most well-known difference between the guiding center motion and gyrocenter motion is the polarization drift motion due to the time-dependent electrical perturbation. We follow Brizard $[12]$ in using the terms 'gyrocenter' and 'guiding center' to distinguish these two 
different coordinate systems.

For the transformation

$$
\bar{Z}^{i}=Z^{i}+G^{i}(Z)
$$

the leading order transformed extended phase space Lagrangian is:[9]

$$
\bar{\gamma}_{E 1}=\gamma_{E 1}-i_{G} \omega_{E 0}+d S=\widehat{\bar{\gamma}}_{E 1}-\bar{H}_{E 1} d \tau
$$

where $\omega_{E 0}=d \gamma_{E 0}, S$ is the gauge function, and $i_{G} \omega_{E 0}$ is the interior product between the vector field $G$ and the two form $\omega_{E 0}$. There are several ways to make $\widehat{\bar{\gamma}}_{E}$ and $\bar{H}_{E} d \tau$ gyrophase independent. We will choose $\boldsymbol{G}$ and $S$ such that there is no perturbation on the symplectic structure,

$$
\widehat{\bar{\gamma}}_{E 1}=0
$$

This will effectively transfer the perturbation into the Hamiltonian. Since we choose not to change the time variable $t, G^{t}=0$. Other components of $\boldsymbol{G}$ are solved for from $\widehat{\bar{\gamma}}_{E 1}=0$.

$$
\begin{aligned}
\boldsymbol{G} & =-\frac{c}{e B_{\|}^{*}} \boldsymbol{b} \times\left(\frac{e}{c} \boldsymbol{A}_{1}+\nabla S\right)-\frac{\boldsymbol{B}^{*}}{m B_{\|}^{*}} \frac{\partial S_{1}}{\partial U}+O\left(\epsilon_{B}\right), \\
G^{U} & =\frac{\boldsymbol{B}^{*}}{m B_{\|}^{*}} \cdot\left(\frac{e}{c} \boldsymbol{A}_{1}+\nabla S\right)+O\left(\epsilon_{B}\right), \\
G^{\mu} & =\frac{e}{m c}\left(\frac{e}{c} \boldsymbol{A}_{1} \cdot \frac{\partial \boldsymbol{\rho}_{0}}{\partial \xi}+\frac{\partial S}{\partial \xi}\right), \\
G^{\xi} & =-\frac{e}{m c}\left(\frac{e}{c} \boldsymbol{A}_{1} \cdot \frac{\partial \boldsymbol{\rho}_{0}}{\partial \mu}+\frac{\partial S}{\partial \mu}\right)+O\left(\epsilon_{B}\right), \\
G^{w} & =-\frac{\partial S}{\partial t} .
\end{aligned}
$$


The transformed Hamiltonian is

$\bar{H}_{E 1}=H_{E 1}-G^{i} \frac{\partial H_{E 0}}{\partial x^{i}}+G^{w}=e \phi_{1}\left(\overline{\boldsymbol{X}}+\boldsymbol{\rho}_{0}, t\right)-\frac{e}{c} \boldsymbol{A}_{1}\left(\overline{\boldsymbol{X}}+\boldsymbol{\rho}_{0}, t\right) \cdot\left\{\overline{\boldsymbol{X}}+\boldsymbol{\rho}_{0}, H_{E 0}\right\}-\left\{S, H_{E 0}\right\}$,

in which

$$
\left\{\overline{\boldsymbol{X}}+\boldsymbol{\rho}_{0}, H_{E 0}\right\}=\boldsymbol{v}+\boldsymbol{v}_{d}+O\left(\epsilon_{B}\right) .
$$

In the calculation related to the gyrocenter transformation, we will only keep the lowest order in terms of $\epsilon_{B}$, because the background FLR effects normally are not important.

We choose

$$
\bar{H}_{E 1}=e\left\langle\phi_{1}\left(\overline{\boldsymbol{X}}+\boldsymbol{\rho}_{0}, t\right)-\boldsymbol{v} \cdot \frac{e}{c} \boldsymbol{A}_{1}\left(\overline{\boldsymbol{X}}+\boldsymbol{\rho}_{0}, t\right)\right\rangle
$$

where $\langle>$ represents the gyrophase averaging operation. This leads to the equation determining the gauge function $S$ :

$$
\begin{aligned}
\left\{S, H_{E 0}\right\} & =\Omega \frac{\partial S}{\partial \xi}+\frac{\partial S}{\partial t}+\frac{\partial S}{\partial \overline{\boldsymbol{X}}} \cdot\left\{\boldsymbol{X}, H_{E 0}\right\}+\frac{\partial S}{\partial U}\left\{U, H_{E 0}\right\} \\
& =e \widetilde{\phi_{1}}\left(\overline{\boldsymbol{X}}+\boldsymbol{\rho}_{0}, t\right)-\frac{e}{c} \widetilde{\boldsymbol{v} \cdot \boldsymbol{A}_{1}}\left(\overline{\boldsymbol{X}}+\boldsymbol{\rho}_{0}, t\right)
\end{aligned}
$$

where $\widetilde{\phi}_{1}\left(\overline{\boldsymbol{X}}+\boldsymbol{\rho}_{0}, t\right)$ and $\widetilde{\boldsymbol{v} \cdot \boldsymbol{A}_{1}}\left(\overline{\boldsymbol{X}}+\boldsymbol{\rho}_{0}, t\right)$ are the gyrophase dependent parts of $\phi_{1}\left(\overline{\boldsymbol{X}}+\boldsymbol{\rho}_{0}, t\right)$ and $\boldsymbol{v} \cdot \boldsymbol{A}_{1}\left(\overline{\boldsymbol{X}}+\boldsymbol{\rho}_{0}, t\right)$ respectively.

$$
\begin{aligned}
\widetilde{\phi_{1}}\left(\overline{\boldsymbol{X}}+\boldsymbol{\rho}_{0}, t\right) & =\phi_{1}\left(\overline{\boldsymbol{X}}+\boldsymbol{\rho}_{0}, t\right)-\left\langle\phi_{1}\left(\overline{\boldsymbol{X}}+\boldsymbol{\rho}_{0}, t\right)\right\rangle \\
\widetilde{\boldsymbol{v} \cdot \boldsymbol{A}_{1}}\left(\overline{\boldsymbol{X}}+\boldsymbol{\rho}_{0}, t\right) & =\boldsymbol{v} \cdot \boldsymbol{A}_{1}\left(\overline{\boldsymbol{X}}+\boldsymbol{\rho}_{0}, t\right)-\left\langle\boldsymbol{v} \cdot \boldsymbol{A}_{1}\left(\overline{\boldsymbol{X}}+\boldsymbol{\rho}_{0}, t\right)\right\rangle .
\end{aligned}
$$


To the lowest order,

$$
\Omega \frac{\partial S}{\partial \xi}=e \widetilde{\phi}_{1}\left(\overline{\boldsymbol{X}}+\boldsymbol{\rho}_{0}, t\right)-\frac{e}{c} \widetilde{\boldsymbol{v} \cdot \boldsymbol{A}_{1}}\left(\overline{\boldsymbol{X}}+\boldsymbol{\rho}_{0}, t\right)
$$

Since $\widehat{\bar{\gamma}}_{E 1}=0$, the Poisson bracket in the gyrocenter coordinates is the same as that in the guiding center coordinates, which is give by Equation (3).

Now we are ready to obtain the linear GKE. Unless clarity requires us to use the barred notation, we will drop the bars for the gyrocenter coordinates thereafter. In the gyrocenter coordinates $(\boldsymbol{X}, U, \mu, \xi, w, t)$, the distribution function $F(\boldsymbol{X}, U, \mu, \xi, w, t)$ satisfies the Vlasov equation:

$$
\left\{F, H_{E}\right\}=\frac{\partial F}{\partial t}+\{F, H\}=\frac{\partial F}{\partial t}+\dot{\boldsymbol{X}} \frac{\partial F}{\partial \boldsymbol{X}}+\dot{U} \frac{\partial F}{\partial U}+\dot{\xi} \frac{\partial F}{\partial \xi}=0
$$

We prove that $F$ is gyrophase independent. Let

$$
F=F^{(0)}+\epsilon_{B} F^{(1)}+\epsilon_{B}^{2} F^{(2)}+\ldots
$$

The leading order is:

$$
\frac{\partial F^{(0)}}{\partial \xi}=0
$$

$F^{(0)}$ is gyrophase independent. To the next order:

$$
\frac{\partial F^{(0)}}{\partial t}+\dot{\boldsymbol{X}} \frac{\partial F^{(0)}}{\partial \boldsymbol{X}}+\dot{U} \frac{\partial F^{(0)}}{\partial U}+\dot{\xi} \frac{\partial F^{(1)}}{\partial \xi}=0
$$

Since all the terms except for $\partial F^{(1)} / \partial \xi$ are gyrophase independent, gyrophase averaging this 
equation gives,

$$
\frac{\partial F^{(0)}}{\partial t}+\dot{\boldsymbol{X}} \frac{\partial F^{(0)}}{\partial \boldsymbol{X}}+\dot{U} \frac{\partial F^{(0)}}{\partial U}=0
$$

and therefore

$$
\dot{\xi} \frac{\partial F^{(1)}}{\partial \xi}=0
$$

By the same way, we can prove that $F$ is gyrophase independent to all orders.

The linear GKE in its geometric form (coordinate independent form) can be written as:

$$
\left\{f, H_{E}\right\}+\left\{F_{0}, H_{E 1}\right\}=0
$$

or

$$
\frac{\partial f}{\partial t}+\left\{f, H_{0}\right\}=-\left\{F_{0}, H_{1}\right\}
$$

where

$$
\begin{aligned}
F & =F_{0}+f, \\
H_{0} & =\frac{m U^{2}}{2}+\mu B_{0}, \\
H_{1} & =\left\langle e \phi_{1}\left(\boldsymbol{X}+\boldsymbol{\rho}_{0}, t\right)-\frac{e}{c} \boldsymbol{v} \cdot \boldsymbol{A}_{1}\left(\boldsymbol{X}+\boldsymbol{\rho}_{0}, t\right)\right\rangle .
\end{aligned}
$$


In the coordinates $(\boldsymbol{X}, U, \mu, \xi, w, t)$, the linear GKE is,

$$
\frac{\partial f}{\partial t}+\left(U \boldsymbol{b}+\boldsymbol{v}_{d}\right) \cdot \nabla f-\frac{1}{m} \boldsymbol{b} \cdot \nabla H_{0}=\frac{c}{e B} \boldsymbol{b} \cdot\left(\nabla F_{0} \times \nabla H_{1}\right)-\frac{1}{m} \boldsymbol{b} \cdot\left(\nabla F_{0} \frac{\partial H_{1}}{\partial U}-\nabla H_{1} \frac{\partial F_{0}}{\partial U}\right)
$$

Another set of gyrocenter coordinates $(\boldsymbol{X}, \varepsilon, \mu, \xi, w, t)$ is often used. $\varepsilon$ is the total energy in the unperturbed field, that is

$$
\varepsilon=H_{0}=\frac{m U^{2}}{2}+\mu B_{0}
$$

In this set of gyrocenter coordinates, the linear GKE is:

$$
\frac{\partial f}{\partial t}+\left(U \boldsymbol{b}+\boldsymbol{v}_{d}\right) \cdot \nabla f=\left(\frac{c}{e B} \times \nabla F_{0}\right) \cdot \nabla H_{1}+\frac{\partial F_{0}}{\partial \varepsilon}\left(U \boldsymbol{b}+\boldsymbol{v}_{d}\right) \cdot \nabla H_{1}
$$

An alternative form of this equation is written in term of the nonadiabatic part of $f$,

$$
\begin{gathered}
g=f-H_{1} \frac{\partial F_{0}}{\partial \varepsilon} . \\
\frac{\partial f}{\partial t}+\left(U \boldsymbol{b}+\boldsymbol{v}_{d}\right) \cdot \nabla f=\left(\frac{c}{e B} \times \nabla F_{0} \cdot \nabla-\frac{\partial F_{0}}{\partial \varepsilon} \frac{\partial}{\partial t}\right) H_{1} .
\end{gathered}
$$

The gyrokinetic Maxwell equations are as important as the GKE itself. The differences between different versions of the GKE can be usually resolved when the corresponding gyrokinetic Maxwell equations are taken into account in appropriate coordinate systems. 
The Poisson equation is

$$
-\nabla^{2} \phi(\boldsymbol{r}, t)=4 \pi \sum_{j} e \int d^{3} \boldsymbol{v} f(\boldsymbol{r}, t, \boldsymbol{v})+\frac{1}{c} \frac{\partial}{\partial t} \nabla \cdot \boldsymbol{A}(\boldsymbol{r}, t)
$$

where

$$
\int d^{3} \boldsymbol{v} f(\boldsymbol{r}, t, \boldsymbol{v})=\int d^{6} \boldsymbol{Z}\left[T_{G Y}^{*} f\right](\boldsymbol{Z}, t) \delta\left(T_{G C *}^{-1} \boldsymbol{X}-\boldsymbol{r}\right) .
$$

Ampere's law is

$$
\nabla \times(\nabla \times \boldsymbol{A}(\boldsymbol{r}, t))=\frac{4 \pi}{c} \sum_{j} e \int d^{3} \boldsymbol{v} \boldsymbol{v} f(\boldsymbol{r}, t, \boldsymbol{v})
$$

where

$$
\int d^{3} \boldsymbol{v} \boldsymbol{v} f(\boldsymbol{r}, t, \boldsymbol{v})=\int d^{6} \boldsymbol{Z}\left[\boldsymbol{V}_{g c}(\boldsymbol{Z})\right]\left[T_{G Y}^{*} f(\boldsymbol{Z}, t)\right] \delta\left(T_{G C *}^{-1} \boldsymbol{X}-\boldsymbol{r}\right)
$$

In above equations, $d^{6} \boldsymbol{Z}$ is understood to be $\left(B_{\|}^{*} / m\right) d^{3} \boldsymbol{X} d U d \mu d \xi$. $T_{G Y}^{*}$ is the pull-back transformation, which transforms the perturbed distribution $f$ in the gyrocenter coordinates into that in the guiding center coordinates. $T_{G C *}^{-1}$ is the inverse of the push-forward $T_{G C *}$ that transforms the particle physical coordinates $(\boldsymbol{r}, \boldsymbol{v}, t)$ into the guiding center coordinates. We assume $T_{G C}^{*}, T_{G C *}, T_{G Y}^{*}$ and $T_{G Y *}$ are one-one onto (bijective). Generally for a macroscopic quantity $Q(\boldsymbol{r})$ in the particle coordinates, we have

$$
Q(\boldsymbol{r})=\int Q(\boldsymbol{r}, \boldsymbol{v}) f_{P H}(\boldsymbol{r}, \boldsymbol{v}) d^{3} \boldsymbol{v}=\int \delta(\boldsymbol{x}-\boldsymbol{r}) Q(\boldsymbol{z}) f_{P H}(\boldsymbol{z}) d^{6} \boldsymbol{z}
$$


In the guiding center coordinates $\boldsymbol{Z}=(\boldsymbol{X}, U, \mu, \xi)$,

$$
Q(\boldsymbol{r})=\int\left[T_{G C}^{*-1} Q\right](\boldsymbol{Z}) f_{G C}(\boldsymbol{Z}) \delta\left(T_{G C *}^{-1}-\boldsymbol{r}\right) d^{6} \boldsymbol{Z}
$$

Treating $\boldsymbol{Z}$ in the above equation as a dummy variable, and replacing it by the gyrocenter coordinates $\overline{\boldsymbol{Z}}=(\overline{\boldsymbol{X}}, \bar{U}, \bar{\mu}, \bar{\xi}, \bar{w}, \bar{t})$, we get,

$$
Q(\boldsymbol{r})=\int\left[T_{G C}^{*-1} Q\right](\overline{\boldsymbol{Z}})\left[T_{G Y}^{*} f_{G Y}\right](\overline{\boldsymbol{Z}}) \delta\left(T_{G C *}^{-1} \overline{\boldsymbol{X}}-\boldsymbol{r}\right) d^{6} \overline{\boldsymbol{Z}}
$$

The pull-back transformation from the gyrocenter coordinates to the guiding center coordinates is easily obtained from the expression for $G$ given by Equation (14),

$$
\begin{aligned}
T_{G Y}^{*} F & =F+L_{\boldsymbol{G}} F \\
& =-\frac{\boldsymbol{b}}{B} \times \boldsymbol{A}_{1} \cdot \nabla F+\frac{e}{m c} \boldsymbol{b} \cdot \boldsymbol{A}_{1} \frac{\partial F}{\partial U}+\frac{e}{m c}\left[\frac{e}{c} \boldsymbol{A}_{1} \cdot \frac{\partial \boldsymbol{\rho}_{0}}{\partial \xi}+\frac{\partial S}{\partial \xi}\right] \frac{\partial F}{\partial \mu}+O\left(\epsilon_{B}\right),
\end{aligned}
$$

where $L_{G} F$ represents the Lie derivative of $F$ with respect to the vector field $\boldsymbol{G}$.

We will use $\boldsymbol{A}$ and $\phi$ to notate the perturbed field thereafter; the subscript "1" will be dropped.

\section{Arbitrary wave-length electromagnetic gyrokinetic system for shear Alfvén physics}

As discussed before, the most influential factors which solely define the characteristics of long wavelength modes are the background inhomogeneities, including the inhomogeneities of equilibrium temperature, density, magnetic field, and current. We need to describe these 
inhomogeneities properly in the kinetic context in order to develop a successful kinetic theory for arbitrary wavelength electromagnetic modes. At first thought, one would suggest a simple solution: express all the background inhomogeneities by an inhomogeneous and anisotropic equilibrium distribution function, and carry out the rest of the process straightforwardly. But for more realistic systems, such as a tokamak, this simple solution won't work out easily. The reason is that when we put all the physical effects, the background inhomogeneities and the kinetic effects, into the distribution function, they entangle together in such a complex way that the problem is not tractable anymore.

Another method is necessary. To proceed, let's observe some basic facts associated with the anisotropic distribution function

$$
F_{j}=\frac{n_{j}(r)}{\left[2 \pi T_{j}(r) / m_{j}\right]^{3 / 2}} \exp \left[-\frac{m_{j}\left[v_{\perp}^{2}+\left(v_{\|}-u_{j}\right)^{2}\right]}{2 T_{j}(r)}\right] .
$$

The equilibrium current produced by the inhomogeneity of the $B$ field can be separated out from the unperturbed distribution function by taking the first moment,

$$
\int \boldsymbol{v} F_{j} d^{3} v=n_{j} \boldsymbol{u}_{j}
$$

which suggests singling out the equilibrium current in deriving the GKM equation. Another fact is that the anisotropy of the equilibrium distribution function turns out to be weak. For example, to create the toroidal current for the poloidal field in a tokamak,

$$
J=e n u \sim \frac{c}{4 \pi} \nabla \times B_{p}, \quad u \sim \frac{c B_{p}}{4 \pi e n a} .
$$


For standard tokamak parameters,

$$
\frac{u^{2}}{v_{t h}^{2}} \sim 2 \frac{c^{2}}{v_{t h}^{2}} \frac{1}{\beta_{p}} \frac{\lambda_{D}^{2}}{a^{2}} \sim \begin{cases}10^{-8}, & \text { electrons } \\ 10^{-5}, & \text { ions. }\end{cases}
$$

This estimate suggests that we can almost assume the unperturbed distribution to be isotropic. But we have to assume an anisotropic distribution, since the equilibrium inhomogeneities can't be ignored. The answer to this seeming paradox is that the bulk of the plasma doesn't contribute to the magnetic field inhomogeneity; the anisotropic part of the plasma is the only source for the magnetic field inhomogeneity although its population is extremely small. Hence we can't throw away the anisotropic component for this reason. For other effects, like perturbed pressure effects, collision effects, and Landau damping, where the contribution from the isotropic part is non-vanishing, the contribution from the anisotropic part can be ignored completely. Our methodology here is to separate out the terms related to the anisotropy in velocity space during the process of deriving the GKM, after which the unperturbed distribution function will be assumed to be isotropic.

Most of the important non-electrostatic long wavelength eigenmodes in tokamak geometry are shear Alfvén waves, that is, the parallel magnetic perturbation is much smaller than the perpendicular magnetic perturbation. Kink modes and TAE modes fall into this category. In a homogeneous medium, the shear Alfvén wave is the branch with the dispersion relation $\omega^{2}=k_{\|}^{2} v_{A}^{2}$. It has several characteristics:

- $B_{1} \perp\left(\boldsymbol{k} \times B_{0}\right), \boldsymbol{v}_{1} \perp\left(\boldsymbol{k} \times \boldsymbol{B}_{0}\right), \boldsymbol{B}_{1 \|}=0$, and $\boldsymbol{v}_{1 \|}=0$.

- $\rho_{1}=0, p_{1}=0$, and $\nabla \cdot \boldsymbol{v}_{1}=0$.

For the shear Alfvén wave in an inhomogeneous plasma, these properties are not all true. 
We will restrict ourselves to large aspect ratio tokamak geometries. We define the shear Alfvén modes in a large aspect ratio tokamak as the modes with

$$
\boldsymbol{A}_{\perp}=0 .
$$

This definition is consistent with the normal meaning of shear Alfvén wave, because from

$$
\boldsymbol{B}_{1}=\nabla \times \boldsymbol{A}=\nabla \times\left(A_{\|} \boldsymbol{b}_{0}\right)=\nabla A_{\|} \times \boldsymbol{b}_{0}+A_{\|} \nabla \times \boldsymbol{b}_{0}
$$

we get the estimate

$$
\boldsymbol{B}_{1 \perp} \sim \boldsymbol{b}_{0} \times \nabla \times \boldsymbol{b}_{0} A_{\|} \sim \frac{A_{\|}}{R} \ll \boldsymbol{B}_{1 \|} \sim \frac{A_{\|}}{r}
$$

We also have $\nabla \cdot \xi \approx 0$. From

$$
\boldsymbol{B}_{1}=\nabla \times\left(\boldsymbol{\xi} \times \boldsymbol{B}_{0}\right)=-\boldsymbol{B}_{0} \nabla \cdot \boldsymbol{\xi}+\left(\boldsymbol{B}_{0} \cdot \nabla\right) \boldsymbol{\xi}-(\boldsymbol{\xi} \cdot \nabla) \boldsymbol{B}_{0}
$$

and $\boldsymbol{B}_{1 \|} \ll \boldsymbol{B}_{1 \perp}$, it is easy to observe

$$
B_{1 \|} \sim-B_{0||}(\nabla \cdot \boldsymbol{\xi})+i B_{0} k_{\| \mid} \xi_{\|}=B_{0||} \boldsymbol{k}_{\perp} \cdot \boldsymbol{\xi}_{\perp} \ll k_{\perp} \xi_{\perp} B_{0}
$$

That is $\boldsymbol{k}_{\perp} \cdot \boldsymbol{\xi}_{\perp} \ll k_{\perp} \xi_{\perp} \sim k \xi$.

Other shear Alfvén characteristics in a homogeneous medium generally are not valid in tokamak geometries. However, the pressure perturbation sometimes can be treated as a small correction by the virtue of the low $\beta$ assumption. This is obvious from the motion 
equation:

$$
\begin{gathered}
\rho_{0} \frac{\partial \boldsymbol{v}}{\partial t}=\frac{1}{c} \boldsymbol{J}_{1} \times \boldsymbol{B}_{0}+\frac{1}{c} \boldsymbol{J}_{0} \times \boldsymbol{B}_{1}-\nabla p_{1} . \\
\frac{1}{c} \boldsymbol{J}_{1} \times \boldsymbol{B}_{0} \sim 4 \pi B_{0}^{2} \frac{\xi}{r^{2}} \gg \nabla p_{1} \sim \nabla \cdot \boldsymbol{\xi} \nabla p_{0} \sim \frac{\xi}{r^{2}} p_{0} .
\end{gathered}
$$

Overall, the shear Alfvén waves that we will study in the large aspect ratio low $\beta$ tokamaks are those almost incompressible eigenmodes with zero perpendicular vector potential perturbations, small parallel magnetic perturbations, and small pressure perturbations.

For shear Alfvén physics,

$$
\boldsymbol{A}=A_{\|} \boldsymbol{b}_{0}
$$

We need three equations to complete the system. Besides the GKE, the gyrokinetic Poisson equation and the gyrokinetic parallel Ampere's law are used. Carrying out the gyrophase averaging, we obtain

$$
H_{1}=\frac{e}{m} J_{0} \phi(\boldsymbol{X})-\frac{e}{m c} J_{0} U A_{\|}(\boldsymbol{X}) .
$$

In this equation and other equations appearing latter, $J_{0}\left(v_{\perp} \nabla_{\perp} / i \Omega\right)$ should be viewed as a symbol for the differential operator defined below:

$$
J_{0}\left(\frac{v_{\perp} \nabla_{\perp}}{i \Omega}\right)=1+\frac{v_{\perp}^{2} \nabla_{\perp}^{2}}{4 \Omega}+\ldots
$$

To derive the explicit forms of the gyrokinetic Maxwell's equations, we first look at the 
pull-back transformation $T_{G Y}^{*} f$. The special form of Equation (20) for shear Alfvén modes is

$$
\Omega \frac{\partial S}{\partial \xi}=\frac{e}{\Omega} \rho_{0} \cdot\left[\nabla \phi(\boldsymbol{X}, t)-\frac{1}{c} U \nabla A_{\|}(\boldsymbol{X}, t)\right]
$$

Using Equation (42), we get the pull-back transformation for shear Alfvén modes,

$$
T_{G Y}^{*} F=F+\frac{e}{m c} A_{\|}\left(\boldsymbol{X}+\boldsymbol{\rho}_{0}, t\right) \frac{\partial F}{\partial U}+\frac{e}{B}\left[\widetilde{\phi}\left(\boldsymbol{X}+\boldsymbol{\rho}_{0}, t\right)-\frac{1}{c} \widehat{U A} \|\left(\boldsymbol{X}+\boldsymbol{\rho}_{0}, t\right)\right] \frac{\partial F}{\partial \mu} .
$$

The perturbed density, perturbed flow, and perturbed current can be derived from the general form of Equation(41).

$$
\begin{aligned}
n(\boldsymbol{r})= & \int\left[T_{G Y}^{*} f\right](\boldsymbol{Z}) \delta\left(\boldsymbol{X}+\boldsymbol{\rho}_{0}-\boldsymbol{r}\right) d^{6} \boldsymbol{Z} \\
= & \int f(\boldsymbol{Z}) \delta(\boldsymbol{X}-\boldsymbol{r}) d^{6} \boldsymbol{Z}+\int\left[\delta\left(\boldsymbol{X}+\boldsymbol{\rho}_{0}-\boldsymbol{r}\right)-\delta(\boldsymbol{X}-\boldsymbol{r})\right] f(\boldsymbol{Z}) d^{6} \boldsymbol{Z} \\
& +\int \delta\left(\boldsymbol{X}+\boldsymbol{\rho}_{0}-\boldsymbol{r}\right)\left\{\frac{e}{m c} A_{\|}\left(\boldsymbol{X}+\boldsymbol{\rho}_{0}, t\right) \frac{\partial F}{\partial U}\right. \\
& \left.+\frac{e}{B}\left[\widetilde{\phi}\left(\boldsymbol{X}+\boldsymbol{\rho}_{0}, t\right)-\frac{1}{c} \widehat{U A_{\|}}\left(\boldsymbol{X}+\boldsymbol{\rho}_{0}, t\right)\right] \frac{\partial F}{\partial \mu}\right\} d^{6} \boldsymbol{Z} .
\end{aligned}
$$

The physical meaning of this equation is clear. The perturbed density in particle coordinates consists of three parts, the perturbed density in gyrocenter coordinates, the guiding center residue, and the gyrocenter residue. The guiding center residue is related to the equilibrium FLR effect and thus can be ignored. After some lengthy algebra,

$$
n_{1}(\boldsymbol{r}, t)=\int f(\boldsymbol{r}, U, \mu, t) d^{3} \boldsymbol{v}+\frac{e}{m} \nabla_{\perp} \frac{n_{0}}{\Omega^{2}} \nabla \phi(\boldsymbol{r}, t)+\frac{3}{4} \frac{e v_{t}^{2} n_{0}}{m \Omega^{4}} \nabla_{\perp}^{4} \phi(\boldsymbol{r}, t),
$$


where $d^{3} \boldsymbol{v}=2 \pi(B / m) d U d \mu$. For the perturbed parallel flow,

$$
\begin{aligned}
n_{0} V_{\| 1}(\boldsymbol{r})= & \int U\left[T_{G Y}^{*} f\right](\boldsymbol{Z}) \delta\left(\boldsymbol{X}+\boldsymbol{\rho}_{0}-\boldsymbol{r}\right) d^{6} \boldsymbol{Z} \\
& \int U f(\boldsymbol{Z}) \delta(\boldsymbol{X}-\boldsymbol{r}) d^{6} \boldsymbol{Z}+\int\left[\delta\left(\boldsymbol{X}+\boldsymbol{\rho}_{0}-\boldsymbol{r}\right)-\delta(\boldsymbol{X}-\boldsymbol{r})\right] f(\boldsymbol{Z}) d^{6} \boldsymbol{Z} \\
& +\int U \delta\left(\boldsymbol{X}+\boldsymbol{\rho}_{0}-\boldsymbol{r}\right)\left\{\frac{e}{m c} A_{\|}\left(\boldsymbol{X}+\boldsymbol{\rho}_{0}, t\right) \frac{\partial F}{\partial U}\right. \\
& \left.+\frac{e}{B}\left[\tilde{\phi}\left(\boldsymbol{X}+\boldsymbol{\rho}_{0}, t\right)-\frac{1}{c} \widehat{U A_{\|}}\left(\boldsymbol{X}+\boldsymbol{\rho}_{0}, t\right)\right] \frac{\partial F}{\partial \mu}\right\} d^{6} \boldsymbol{Z} .
\end{aligned}
$$

Again, the algebra here is straightforward but involved. The final result is:

$$
n_{0} V_{\| 1}(\boldsymbol{r}, t)=\int U f(\boldsymbol{r}, U, \mu, t) d^{3} \boldsymbol{v}+\int \frac{e}{m c}\left\langle U A_{\|}\left(\boldsymbol{r}+\boldsymbol{\rho}_{0}\right)\right\rangle \frac{\partial F}{\partial U} 2 \pi \frac{B}{m} d \mu d U+\frac{e n_{0} v_{t}^{2}}{2 m c \Omega^{2}} \nabla_{\perp}^{2} A_{\|} .
$$

Therefore, the quasi-neutrality condition is

$$
\sum_{j} e\left[\int f d^{3} \boldsymbol{v}+\frac{e}{m} \nabla_{\perp} \frac{n}{\Omega^{2}} \nabla_{\perp} \phi+\frac{3 e}{4 m} \frac{v_{t}^{2}}{\Omega^{2}} \frac{n}{\Omega} \nabla_{\perp}^{4} \phi\right]=0
$$

and parallel Ampere's law is

$$
[\nabla \times \nabla \times \boldsymbol{A}]_{\|}=\frac{4 \pi}{c} \sum_{j} e \int U\left(f+\frac{\partial F_{0}}{\partial E} \frac{e}{m c}\left\langle U A_{\|}\right\rangle\right) d^{3} \boldsymbol{v}+\frac{4 \pi}{c} \frac{e^{2} n_{0} v_{t}^{2}}{2 m c \Omega^{2}} \nabla_{\perp}^{2} A_{\|} .
$$

In the above equations, the spatial variable is the particle coordinate $\boldsymbol{r}$. However $\boldsymbol{r}$ is a dummy variable. What matters is the functional forms. We can replace $\boldsymbol{r}$ by the spatial coordinate of the gyrocenter coordinate $\boldsymbol{Z}$. The Equations (59) and (60) will be referred to as the gyrokinetic quasineutrality condition and the gyrokinetic parallel Ampere's law respectively. 
Instead of using the parallel Ampere's law directly, we usually combine it with the 0th moment equation of the GKE to get the gyrokinetic moment equation (GKM) and use this as the third equation in our equation system. This equation is often referred as the GKM , in spite of the fact that it is distinct from the gyrokinetic equation because Ampere's law has been utilized to derive it. We use the linear GKE in $(\boldsymbol{X}, U, \mu, \xi)$, Equation (30). The 0th moment of it is

$$
\begin{aligned}
\frac{\partial}{\partial t} & \int f d^{3} \boldsymbol{v}+\int \boldsymbol{v}_{d} \cdot \nabla f d^{3} \boldsymbol{v}+\int U \boldsymbol{b} \cdot \nabla f d^{3} \boldsymbol{v}-\frac{1}{m} \int \boldsymbol{b} \cdot \mu \nabla B \frac{\partial f}{\partial U} d^{3} \boldsymbol{v} \\
& =\frac{c \boldsymbol{b}}{e B} \cdot \int \nabla F_{0} \times \nabla H_{1} d^{3} \boldsymbol{v}-\frac{\boldsymbol{b}}{m} \cdot \int \frac{\partial H_{1} \nabla F_{0}}{\partial U} d^{3} \boldsymbol{v}+\frac{\boldsymbol{b}}{m} \cdot \int \nabla\left(H_{1} \frac{\partial F_{0}}{\partial U}\right) d^{3} \boldsymbol{v}
\end{aligned}
$$

It is obvious that the 4 th term on the left hand side and the 2 nd term on the right hand side vanish. Applying $\sum_{j} e_{j}$, we have

$$
\begin{array}{r}
\sum_{j} e \frac{\partial}{\partial t} \int f d^{3} \boldsymbol{v}+\sum_{j} e \int\left[U \boldsymbol{b} \cdot \nabla f-\boldsymbol{b} \cdot \nabla\left(\frac{H_{1}}{m} \frac{\partial F}{\partial U}\right)\right] d^{3} \boldsymbol{v} \\
+\sum_{j} \frac{\boldsymbol{c} \boldsymbol{b}}{B} \cdot \int \nabla F \times \nabla H_{1} d^{3} \boldsymbol{v}+\sum_{j} e \boldsymbol{v}_{d} \cdot \nabla f d^{3} \boldsymbol{v}=0 .
\end{array}
$$

Using the quasineutrality condition, Equation (59), we replace the first term by

$$
-\sum_{j} \frac{\partial}{\partial t}\left(\frac{e}{m} \nabla_{\perp} \frac{n}{\Omega^{2}} \nabla_{\perp} \phi+\frac{3 e}{4 m} \frac{v_{t}^{2}}{\Omega^{2}} \frac{n}{\Omega} \nabla_{\perp}^{4} \phi\right) .
$$


For the second term, we have

$$
\begin{aligned}
\sum_{j} e & \int\left[\boldsymbol{b} \cdot \nabla f-\boldsymbol{b} \cdot \nabla\left(\frac{H_{1}}{m} \frac{\partial F}{\partial U}\right)\right] \boldsymbol{d}^{3} \boldsymbol{v} \\
& =\sum_{j} e \int \boldsymbol{b} \cdot \nabla\left(U f-\frac{H_{1}}{m} \frac{\partial F}{\partial U}\right) d^{3} \boldsymbol{v} \\
& =\sum_{j} \boldsymbol{B} \cdot \nabla \frac{1}{B} \int\left(U f-\frac{H_{1}}{m} \frac{\partial F}{\partial U}\right) \frac{B}{m} 2 \pi d \mu d U \\
& =\boldsymbol{B} \cdot \nabla\left\{\frac{1}{B} \sum_{j} \int e\left[U f+\frac{e}{m c}\left\langle U A_{\|}\right\rangle \frac{\partial F}{\partial U}\right] 2 \pi B d \mu d U\right\} \\
& =\boldsymbol{B} \cdot \nabla\left\{\frac{1}{B}\left[\frac{c}{4 \pi}\left(\nabla \times \nabla \times \boldsymbol{A}_{\|}\right)_{\|}-\sum_{j} \frac{e^{2} n_{0}}{m c} \frac{v_{t}^{2}}{2 \Omega^{2}} \nabla_{\perp}^{2} A_{\|}\right]\right\} .
\end{aligned}
$$

We note that only in $(\boldsymbol{X}, U, \mu, \xi)$ coordinates can we freely move $U$ in and out of $\nabla$. But we should not move $\boldsymbol{b}$ or $B$ in and out $\nabla$. This is important for arbitrary wavelength modes, for which the equilibrium inhomogeneities are crucial. It will later be clear that this accuracy enables us to exactly recover the ideal MHD equation from our gyrokinetic system. For short wavelength modes, the background variations are normally ignored, and $B, n_{0}$, and $\mathbf{b}$ can be brought in and out of $\nabla$ when necessary.

After some calculation, the third term is

$$
\left(\boldsymbol{b} \times \nabla A_{\|}\right) \cdot \nabla \frac{j_{0 \|}}{B}+\sum_{j} \boldsymbol{c} \boldsymbol{b} \times \nabla \frac{n_{0} v_{t}^{2}}{2 B_{0} \Omega^{2}} \cdot \nabla \nabla_{\perp}^{2} \phi .
$$

As before, no approximations regarding the equilibrium inhomogeneities are made in this calculation.

Finally, the GKM is: 


$$
\begin{aligned}
- & \frac{\partial}{\partial t}\left[\frac{c^{2}}{4 \pi} \nabla \cdot\left(\frac{1}{V_{A}^{2}} \nabla_{\perp} \phi\right)\right]+\frac{c}{4 \pi}\left(\boldsymbol{B}_{0} \cdot \nabla\right) \frac{(\nabla \times \nabla \times \boldsymbol{A}) \cdot \boldsymbol{B}_{0}}{B_{0}^{2}}+\left(\nabla A_{\|} \times \boldsymbol{b}_{0}\right) \cdot \nabla \frac{j_{0 \|}}{B_{0}} \\
= & -\sum_{j} \int\left(e \boldsymbol{v}_{d} \cdot \nabla f\right)_{j} d^{3} v+\sum_{j} \frac{1}{4 \pi} \frac{c^{2}}{v_{A}^{2}}\left(\frac{3 v_{t}^{2}}{4 \Omega^{2}}\right)_{j} \nabla_{\perp}^{4} \frac{\partial \phi}{\partial t} \\
& +\boldsymbol{B} \cdot \nabla\left[\frac{1}{B} \sum_{j}\left(\frac{e^{2} n}{m c} \frac{v_{t}^{2}}{2 \Omega^{2}}\right)_{j} \nabla_{\perp}^{2} A_{\|}\right]+\boldsymbol{b} \times \sum_{j} \nabla\left(\frac{c e n_{0} v_{t}^{2}}{2 B_{0} \Omega^{2}}\right)_{j} \cdot \nabla \nabla_{\perp}^{2} \phi,
\end{aligned}
$$

where the $\int e \boldsymbol{v}_{d} \cdot \nabla f d^{3} v$ is evaluated in $(\boldsymbol{X}, U, \mu, \xi)$ coordinates. In $(\boldsymbol{X}, \varepsilon, \mu, \xi)$ coordinates, it should be replaced by $\int\left(\boldsymbol{v}_{d} \cdot \nabla f+\boldsymbol{v}_{d} \cdot \nabla B \partial f / \partial \varepsilon \mu\right) d^{3} \boldsymbol{v}$. However, in a low $\beta$ plasma,

$$
\int\left(\boldsymbol{v}_{d} \cdot \nabla f+\boldsymbol{v}_{d} \cdot \nabla B \frac{\partial f}{\partial \varepsilon} \mu\right) d^{3} \boldsymbol{v} \approx \int \boldsymbol{v}_{d} \cdot \nabla f d^{3} \boldsymbol{v}
$$

because

$$
\boldsymbol{v}_{d} \approx-\frac{c m\left(v_{\|}^{2}+v_{\perp}^{2} / 2\right)}{e B^{3}} \nabla B \times B .
$$

We will introduce another field variable $\psi_{\|}$to replace $A_{\|} \cdot \psi_{\|}$is defined by

$$
A_{\|} \equiv \frac{c}{i \omega}\left(\nabla \psi_{\|}\right)_{\|}
$$

It is easy to solve for $f$ in $(\boldsymbol{X}, \varepsilon, \mu, \xi)$ coordinates. The non-adiabatic part of the perturbed distribution functions $g$ is solved for in terms of $\phi$ and $\psi_{\|}$from the gyro-kinetic equation (34) by integrating along the characteristic lines. The formal solution is given as:

$$
g=\int_{-\infty}^{t} d t^{\prime}\left\{\left(\frac{\boldsymbol{b}_{\mathbf{0}}}{\Omega} \times \nabla F_{0} \cdot \nabla-\frac{\partial F_{0}}{\partial E} \frac{\partial}{\partial t}\right) H_{1}\right\}
$$


In conclusion, our gyrokinetic system for the arbitrary wavelength shear Alfvén modes consists of three equations, the gyrokinetic equation, Equation(34), the gyrokinetic quasineutrality condition, Equation(59), and the gyrokinetic moment equation, Equation(64).

\section{Comparison and Recovery}

Two simplified versions of our GKM have been derived before to study kinetically TAE related problems. The first one is the equation derived by Rosenbluth and Rutherford; [23] this equation was used by Fu and Van Dam [14] as the starting equation for their kinetic Alfvén modes. Though some inhomogeneities are kept to give the desired result, most of the important inhomogeneities in toroidal geometry, which would affect the final result, were left out. Being aware of this shortcoming, Berk, et al derived a new equation for the same purpose from ideal MHD theory; [24] then they replaced the pressure perturbation term by a kinetic counterpart such that the crucial kinetic effects are able to be picked up. Clearly this is the standard hybrid kinetic-MHD treatment, which is not always selfconsistent.

The advantage of our fully gyrokinetic formalism and the GKM equation is apparent by comparison. First, our selfconsistent, fully kinetic approach is valid for general inhomogeneous plasmas, and all the important physical factors are captured. Therefore, it is capable of delineating the fine structure of these long wavelength modes we are interested in. As we can see from the left hand side of the GKM, the kink terms are more detailed than those in References [23] and [14], and also the right hand side, in addition to being selfconsistent, has kinetic terms that are more comprehensive than those in References [23],[14], and [24].

The kink mode is harder to describe because it appears in the order of $O\left(\epsilon^{2}\right)$, while the TAE comes out in the order of $O(\epsilon)$. Most alternative theories, including Strauss's reduced 
MHD,[25] consider physical effects only to the order $O(\epsilon)$, and thus are not capable of providing information order $O\left(\epsilon^{2}\right)$. Specifically, the internal kink modes can not be recovered by these models.[25, 26, 27] Our system can recover TAE modes as well as kink modes in tokamaks. It is the only known alternative approach for the classical yet still important kink instabilities studied before by MHD theory. Moreover, with the ability to examine the interaction between ideal MHD kink modes and kinetic effects, our system will be a key to investigate systematically some bewildering questions in today's fusion plasma physics. Secondly, the physical features captured in our GKM equation are separated. The background inhomogeneities responsible for the TAE modes, the kink instabilities, and other ideal MHD modes is completely isolated in the left hand side; meanwhile the equilibrium pressure effects, the Landau damping effects, and the FLR effects appear on the right hand side of the equation. It is possible to look at each one of them individually. Finally, our formalism is an arbitrary wavelength description. It is able to recover the results for short wavelength modes. We will use this as a benchmark, while concentrating on the long wave length electromagnetic modes and their kinetic modifications.

Now, we show that our GKM can recover the ideal MHD equations. From $\nabla \cdot \boldsymbol{j}_{1}=0$, we get

$$
\left(\boldsymbol{B}_{0} \cdot \nabla\right) \frac{\boldsymbol{j}_{1} \cdot \boldsymbol{B}_{0}}{B_{0}^{2}}+\nabla \cdot \frac{\boldsymbol{B}_{0} \times\left(\boldsymbol{j}_{1} \times \boldsymbol{B}_{0}\right)}{B_{0}^{2}}=0
$$

The linearized motion equation is

$$
\rho_{0} \frac{\partial \boldsymbol{v}}{\partial t}=\frac{1}{c} \boldsymbol{j}_{1} \times \boldsymbol{B}_{0}+\frac{1}{c} \boldsymbol{j}_{0} \times \boldsymbol{B}_{1}-\nabla p_{1}
$$


from which we obtain

$$
c \rho_{0} \frac{\boldsymbol{B}_{0}}{B_{0}^{2}} \times \frac{\partial \boldsymbol{v}}{\partial t}-\frac{\boldsymbol{B}_{0}}{B_{0}^{2}} \times\left(\boldsymbol{j}_{0} \times \boldsymbol{B}_{1}\right)+c \frac{\boldsymbol{B}_{0}}{B_{0}^{2}} \times \nabla p_{1}=\frac{\boldsymbol{B}_{0}}{B_{0}^{2}} \times\left(\boldsymbol{j}_{1} \times \boldsymbol{B}_{0}\right) .
$$

Also, we have the ideal Ohm's Law

$$
\boldsymbol{B}_{0} \times \boldsymbol{v}=c \boldsymbol{E}_{1} .
$$

We combine Equation (69), (71), and(72) to get:

$$
\left(\boldsymbol{B}_{0} \cdot \nabla\right) \frac{\boldsymbol{j}_{1} \cdot \boldsymbol{B}_{0}}{B_{0}^{2}}+\nabla \cdot \frac{c^{2} \rho_{0}}{B_{0}^{2}} \frac{\partial \boldsymbol{E}_{1}}{\partial t}-\nabla \cdot \frac{\boldsymbol{j}_{0}\left(\boldsymbol{B}_{0} \cdot \boldsymbol{B}_{1}\right)-\boldsymbol{B}_{1}\left(\boldsymbol{B}_{0} \cdot \boldsymbol{j}_{0}\right)}{B_{0}^{2}}+c \nabla p_{1} \cdot \nabla \times \frac{\boldsymbol{B}_{0}}{B_{0}^{2}}=0
$$

For shear Alfvén modes, this is

$$
\left(\boldsymbol{B}_{0} \cdot \nabla\right) \frac{\boldsymbol{j}_{1} \cdot \boldsymbol{B}_{0}}{B_{0}^{2}}+i \omega \nabla \cdot \frac{c^{2} \rho_{0}}{B_{0}^{2}} \nabla_{\perp} \phi+\left(\boldsymbol{B}_{1} \cdot \nabla\right) \frac{\boldsymbol{j}_{0} \cdot \boldsymbol{B}_{0}}{B_{0}^{2}}+c \nabla p_{1} \cdot \nabla \times \frac{\boldsymbol{B}_{0}}{B_{0}^{2}}=0
$$

where we have expressed the perturbation field in terms of $\phi$ and $\psi_{\|}$, and $\boldsymbol{B}_{1}=\nabla \times\left(A_{\|} \boldsymbol{b}_{0}\right) \simeq$ $\boldsymbol{B}_{1 \perp} \simeq \nabla A_{\|} \times \boldsymbol{b}_{0}, \boldsymbol{j}_{1}=(c / 4 \pi) \nabla \times \boldsymbol{B}_{1}$. This equation is the ideal MHD eigenequation for shear Alfvén modes in terms of perturbed electromagnetic fields. It can be recovered from our GKM when the FLR effect is neglected and the first term on the left hand side of the GKM is replaced by its ideal MHD counterpart $c \nabla p_{1} \cdot \nabla \times\left(\boldsymbol{B}_{0} / B_{0}^{2}\right)$. The kinetic generalization of ideal MHD is represented by the left hand side of the GKM, i.e., the kinetically generalized pressure perturbation term and the FLR terms. Therefore it is reasonable to expect our kinetic approach to recover all the important MHD results. Indeed, in the simplest limit, we 
have

$$
\begin{aligned}
\sum_{j} e \boldsymbol{v} \cdot \nabla f d^{3} \boldsymbol{v}=\sum_{j} \frac{m c}{B} \boldsymbol{b} \times\left[\frac{v_{\perp}^{2}}{2} \frac{\nabla B}{B}+U^{2} \boldsymbol{b} \cdot \nabla \boldsymbol{b}\right] \cdot \nabla f d^{3} \boldsymbol{v} \\
\quad=\frac{c}{B}\left[\boldsymbol{b} \times \nabla B \cdot \nabla \sum_{j} \int f \mu d^{3} \boldsymbol{v}+\boldsymbol{b} \times \boldsymbol{b} \cdot \nabla \boldsymbol{b} \cdot \nabla \sum_{j} \int f m U^{2} d^{3} \boldsymbol{v}\right] \\
\quad \approx \frac{c}{B} \boldsymbol{b} \times\left(\frac{\nabla B}{B}+\boldsymbol{b} \cdot \nabla \boldsymbol{b}\right) \cdot \nabla p_{1} \\
\quad \approx c\left(\boldsymbol{b} \times \frac{\nabla B}{B^{2}}+\nabla \frac{\times \boldsymbol{b}}{B}\right) \cdot \nabla p_{1} \\
=c \nabla p_{1} \cdot \nabla \times \frac{\boldsymbol{B}}{B^{2}} .
\end{aligned}
$$

\section{Solution methods in tokamak geometry}

In this section, we discuss briefly the solution methods for our equation system. More details will be presented in future publications.

One of the difficulties in solving the basics Equations (34), (59), and (60) is that it is a integral-differential equation system in a $6 \mathrm{D}$ space - 3D configuration space and $3 \mathrm{D}$ velocity space. Though these equations can be studied directly by particle simulation, the linear eigenmode problem can be rigorously projected onto the $3 \mathrm{D}$ configuration space by solving the linear GKE for the perturbed distribution function in terms of field variables, and substituting the solution back into the the quasi-neutrality condition and the GKM. When combined with boundary conditions, an eigenvalue problem is formed.

This method has been successfully applied to the kinetic theory of both short wave length electromagnetic modes and long wavelength electrostatic modes. In the latter case, of course, the quasi-neutrality condition itself will complete the system; the GKM is not used.

When applying the equation system to the geometries of large aspect ratio tokamaks, we encounter another difficulty. As one can imagine, the left hand side of the GKM equation as 
a scalar function of $\psi_{\|}$and $\phi$ is extremely complicated in tokamak geometries. To study the TAE modes kinetically, we have to evaluate this equation to order $O(\epsilon)$. For internal kink modes, it has to be calculated to order $O\left(\epsilon^{2}\right)$. Even assuming circular concentric magnetic surfaces, the total number of terms involved to order $O\left(\epsilon^{2}\right)$ is about 1,500 , which is obviously problematic if calculating by hand.

We have developed a computer algebra package for vector analysis in general coordinate systems, called GVA, in the context of symbolic computation system Mathematica.[18, 28] GVA can perform symbolic vector calculation in any mathematically well defined coordinate system. Asymptotic analysis capability is built into this package, and any analytical result can be expanded as an asymptotic series. With the help of the GVA, we are able to work out the required vector calculation to any order of $\epsilon$ quickly with $100 \%$ accuracy after specifying an equilibrium magnetic field and a coordinate system.

The simplest toroidal model equilibrium assumes circular, concentric flux surfaces, and uses the coordinates $(r, \theta, \zeta)$ shown in the Figure 2.

The magnetic field is given by

$$
\boldsymbol{B}=\frac{B_{0}}{h(\theta)}\left(\boldsymbol{e}_{\zeta}+\frac{r}{q(r) R_{0}} \boldsymbol{e}_{\theta}\right)
$$

where

$$
h(\theta) \equiv 1+\epsilon \cos \theta, \quad \epsilon \equiv \frac{r}{R_{0}}
$$




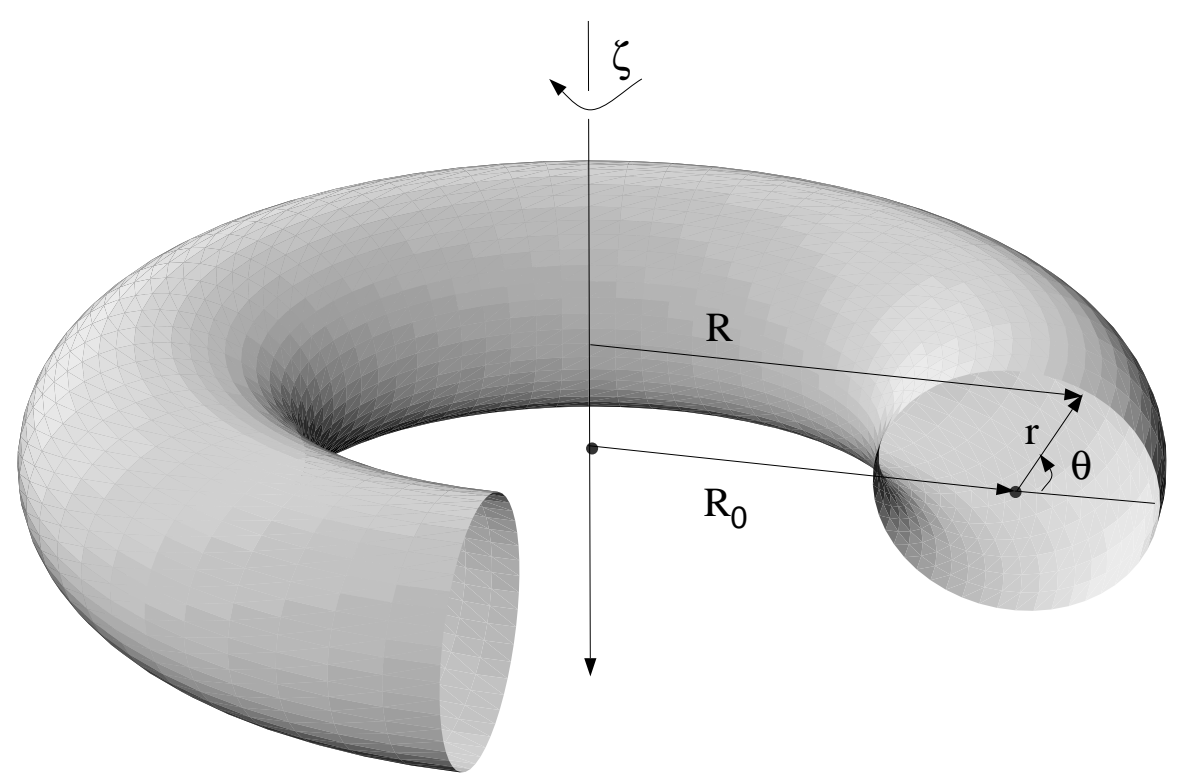

Figure 2: Circular concentric tokamak coordinate system.

Assuming the general 2D expansion[29]

$$
\left(\psi_{\|}, \phi\right)=\sum_{m}\left(\psi_{\| m}(r), \phi_{m}(r)\right) e^{i n \zeta-i m \theta-i \omega t}
$$

let's work out the left hand side of the GKM equation in this coordinate system to the order of $O(\epsilon)^{2}$. There are more than 100 terms.

$$
\begin{aligned}
& -\frac{\partial}{\partial t}\left[\frac{c^{2}}{4 \pi} \nabla \cdot\left(\frac{1}{V_{A}^{2}} \nabla_{\perp} \phi\right)\right]+\frac{c}{4 \pi}\left(\boldsymbol{B}_{0} \cdot \nabla\right) \frac{(\nabla \times \nabla \times \boldsymbol{A}) \cdot \boldsymbol{B}_{0}}{B_{0}^{2}}+(\nabla \times \boldsymbol{A})_{\perp} \cdot \nabla \frac{j_{0 \|}}{B_{0}} \\
& =\sum_{m} e^{i n \zeta-i m \theta-i \omega t}\left(\frac{O_{2}}{R_{0}^{2}}+\frac{O_{3}}{R_{0}^{3}}+\frac{O_{4}}{R_{0}^{4}}\right)
\end{aligned}
$$

$$
O_{3}=D_{1} e^{i \theta}+U_{1} e^{-i \theta}
$$




$$
O_{4}=D_{2} e^{2 i \theta}+U_{2} e^{-2 i \theta}+S
$$

where $O_{2}, O_{3}, O_{4}$, etc are differential operators acting upon every pair of $\psi_{\| m}(r)$ and $\phi_{m}(r)$. $O_{2}, O_{3}$ and $O_{4}$ are $O\left(\epsilon^{0}\right), O\left(\epsilon^{1}\right)$ and $O\left(\epsilon^{2}\right)$ respectively. The $O_{3}$ term can be separated into $D_{1}$ which couples downward by one poloidal harmonic, and $U_{1}$ which couples upward by one poloidal harmonic ; the $O_{4}$ term can be separated into $D_{2}$ which couples downward by two poloidal harmonics, $U_{2}$ which couples upward by two poloidal harmonics, and $S$ which is the self-coupling term. $S$ can be divided further into the self-coupling term from a straight tokamak $S^{s}$ and that from toroidicity $S^{t}$, i.e. $S=S^{s}+S^{t}$. Inside every term, there are terms related to $\psi_{\|}$representing by subscript " $\psi$ " and terms related to $\phi$ representing by subscript " $\phi$ ". For example, $S^{s}=S_{\psi}^{s}+S_{\phi}^{s}$. The expressions for these operators are listed below in Appendix A.

The equation for straight tokamak geometry is a special case and can be recovered when the toroidal coupling terms are set to zero. In this case, poloidal harmonics are decoupled. For each one of them, we have:

$$
\begin{aligned}
& -\frac{\partial}{\partial t}\left[\frac{c^{2}}{4 \pi} \nabla \cdot\left(\frac{1}{V_{A}^{2}} \nabla_{\perp} \phi\right)\right]+\frac{c}{4 \pi}\left(\boldsymbol{B}_{0} \cdot \nabla\right) \frac{(\nabla \times \nabla \times \boldsymbol{A}) \cdot \boldsymbol{B}_{0}}{B_{0}^{2}}+(\nabla \times \boldsymbol{A})_{\perp} \cdot \nabla \frac{j_{0 \|}}{B_{0}} \\
& =\left(\frac{O_{2}}{R_{0}^{2}}+\frac{S^{s}}{R_{0}^{4}}\right) .
\end{aligned}
$$

The equation system in tokamak geometries is generally a coupled system. There are an infinite number of ordinary differential equations coupled together. However, considering the fact that the inverse aspect ratio is a small parameter, we can utilize some perturbation techniques to simplify the system. The important observation is that the coupling between 
different harmonics is proportional to $\epsilon$, as is apparent from Equation (79). The order $O\left(\epsilon^{0}\right)$ term is $O_{2}$, which is decoupled. The order $O\left(\epsilon^{1}\right)$ term is $O_{3}$ which couples to the harmonics higher by one and lower by one. In the order $O\left(\epsilon^{2}\right)$ term, $O_{4}$, we find terms coupled to the harmonics higher by two and lower by two. In other words, the coupling, like $\epsilon$ itself, is a

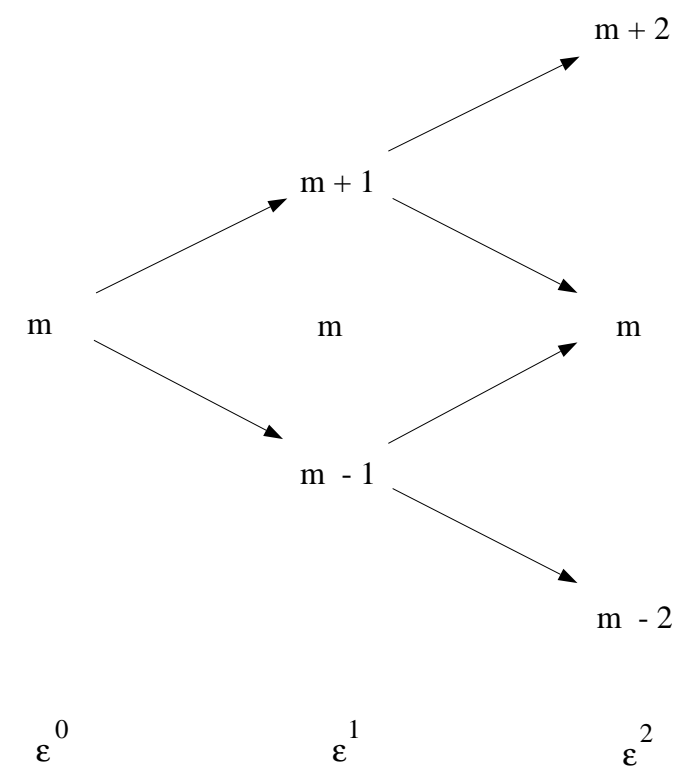

Figure 3: Toroidal coupling diagram.

weak effect. The strongest coupling of a harmonic to other harmonics is in order $O\left(\epsilon^{1}\right)$, and only to its nearest neighbors. The longer the interval between two harmonics, the higher order is the coupling between them. This situation is shown in the coupling diagram in Figure 3.

The method of asymptotic decoupling, that we propose, is based upon this fact. To order $O\left(\epsilon^{0}\right)$, all harmonics are decoupled. Therefore we can pick an eigenmode for $\epsilon=0$, for example $(m, n)=(1,1)$, and ask what the perturbation on this mode is when the small parameter $\epsilon$ is introduced. It is easy to see that to order $O\left(\epsilon^{1}\right)$, two new harmonics appear - the $m-1$ and $m+1$ harmonics. There are only three harmonics in the system now. We 
can solve for the $m-1$ and $m+1$ harmonics and the perturbation on the eigenfrequency and the $m$ harmonic. We can go on to the next order, $O\left(\epsilon^{2}\right)$, to solve for the $m-2$ and $m+2$ harmonics and the second order perturbations on other quantities. This process can be carried out to any order.

One thing we need to realize about this asymptotic decoupling method is that the number of differential equations involved varies as the perturbation process is carrying out. The higher the order, the more the equations. To order $O\left(\epsilon^{n}\right)$, there are $2 n-1$ equations in the system, but only 2 new variables are introduced by each increase of one order.

For those modes whose leading order contains many decoupled harmonics, the asymptotic method will become intractable. Numerical solution is needed. Also, for kinetic effects like trapped particle effects, a numerical code including all the interesting physics are indispensable.

Our gyrokinetic system can be converted into a system of coupled ODEs of the following form:

$$
A_{p m}\left(\begin{array}{c}
\phi_{m}^{\prime \prime} \\
\psi_{\| m}^{\prime \prime}
\end{array}\right)+B_{p m}\left(\begin{array}{c}
\phi_{m}^{\prime} \\
\psi_{\| m}^{\prime}
\end{array}\right)+C_{p m}\left(\begin{array}{c}
\phi_{m} \\
\psi_{\| m}
\end{array}\right)=0
$$

where $A_{p m}, B_{p m}$, and $C_{p m}$ are $2 \times 2$ block matrices whose two rows correspond to the quasi-neutrality condition and the gyrokinetic moment equation respectively, with each block spanning the poloidal harmonics. Here' denotes the radial derivative. Note that $A_{p m}, B_{p m}$, and $C_{p m}$ are functions of $r$ as well as $\omega$.

After truncation to some proper number of poloidal harmonics, this eigenvalue problem is solved numerically using a finite element method in the radial direction. The actual code 
will appear as a version of the two-dimensional kinetic code (KIN-2D) developed over the past 20 years by Tang, Rewoldt, Marchand, and Artun at the Princeton Plasma Physics Laboratory. $[6,7,30,31]$

\section{Two simple applications}

In this section we give two simple applications of our gyrokinetic formalism for arbitrary wavelength electromagnetic modes. As the first application, we derive the local dispersion relation of electrostatic drift waves in slab geometry. Then we recover the classical ideal MHD result of the internal kink mode in a straight tokamak. More interesting applications such as global drift modes, internal kink modes in toroidal geometry, and TAE modes will be covered in future publications.

For the local dispersion relation of electrostatic drift waves in slab geometry, we employ the electrostatic limit of the Equation (32),

$$
\left(\frac{\partial}{\partial t}+U \boldsymbol{b} \cdot \nabla\right) f_{j}-\frac{\nabla \phi \times \boldsymbol{b}}{B} \nabla F_{0 j}-e_{j} \nabla \phi \cdot \boldsymbol{b} U \frac{\partial F_{0 j}}{\partial \varepsilon}=0
$$

whose solution is given by:

$$
f_{j}=F_{0 j} \frac{|e| \phi}{T_{e}}\left[\frac{\omega_{* j}\left(1-3 / 2 \eta_{j}\right)-\omega+k_{\|} U\left(1+\frac{T_{e} e_{j}}{T_{i}|e|}\right)+\omega_{* e} \eta_{j} \frac{\varepsilon}{T_{j}}}{\omega-k_{\|} U}+1\right]
$$

where $\omega_{* j}=\left(k_{\perp} T / m \Omega\right)_{j} d n_{j 0} / d x$ is the diamagnetic drift frequency, and $\eta_{j}=d \ln T_{j} / d \ln n_{j 0}$. 
This solution is substituted in the quasineutrality condition,

$$
\sum_{j} e_{j}\left[\int f_{j}(\boldsymbol{X}, U, \mu, t) 2 \pi B d \mu d U+\frac{e_{j}}{m_{j}} \nabla_{\perp} \frac{n_{j 0}}{\Omega_{j}^{2}} \nabla_{\perp} \phi\right]=0
$$

to derive the dispersion relation. Normally, $F_{0 j}$ can be assumed to be Maxwellian in the gyrocenter coordinates. Then the density response in the gyrocenter coordinates can be expressed in the following familiar form,

$$
\begin{aligned}
\int f_{j}(\boldsymbol{X}, U, \mu, t) 2 \pi d \mu d U= & \frac{|e| \phi}{T_{e}} n_{0 j}\left\{-\frac{T_{e} e_{j}}{T_{j}|e|}-\frac{Z(\xi)}{k_{\|} v_{t}}\left[\omega_{* e}\left(1-1 / 2 \eta_{j}\right)+\omega \frac{T_{e} e_{j}}{T_{j}|e|}\right]\right. \\
& \left.-\frac{\omega_{* e} \eta_{j} \xi}{k_{\|} v_{t}}[1+\xi Z(\xi)]\right\}
\end{aligned}
$$

where $\xi=\omega / k_{\|} v_{t}$. It is sufficient to only keep the gyrocenter residue of ions, because $\Omega_{e}^{2} m_{e} \gg \Omega_{i}^{2} m_{i}$. As usual, electrons are assumed to be hot, that is $\xi_{e} \ll 1$, and ions to be cold, that is, $\xi_{i} \gg 1$. We also assume that $T_{e}=T_{j}$. Working out the algebra straightforwardly, we obtain the dispersion relation,

$$
1-\frac{\omega_{* e}}{\omega}+b_{s}-\frac{1}{2}\left(\frac{k_{\|} C_{s}}{\omega}\right)^{2}\left(1-\frac{\omega_{* p i}}{\omega}\right)=\frac{i \sqrt{\pi}}{k_{\|} v_{t e}}\left[\omega_{* e}\left(1-\eta_{e} / 2\right)-\omega\right],
$$

where $b_{s}=T_{e} k_{\perp}^{2} /\left(m_{i} \Omega_{i}^{2}\right) \phi . \quad b_{s}$ comes directly from the gyrocenter residue which is due to the polarization drift in the perturbed time-dependent electrical field. Without the kinetic correction on the right hand side, it is the well-known fluid result. We emphasize that the appearance of the ion gyrocenter residue in the quasineutrality condition guarantees us a complete recovery of the fluid result.

The second application here is the classical internal kink mode in a straight tokamak. The familiar ideal MHD result from the energy principle can summarized as follows, 


$$
\begin{cases}m^{2} \neq 1 & \text { All modes are stable to } O\left(\epsilon^{0}\right) \\ m^{2}=1 & \begin{cases}q(r=0)>\frac{1}{n} & \text { stable to } O\left(\epsilon^{0}\right) \\ q(r=0)<\frac{1}{n} & \text { neutral to } O\left(\epsilon^{0}\right)\end{cases} \\ & \text { unstable to } O\left(\epsilon^{2}\right)\end{cases}
$$

In the straight tokamak approximation, all poloidal harmonics are decoupled. Our GKM gives:

$$
\frac{O_{2}}{R_{0}^{2}}+\frac{S^{s}}{R_{0}^{4}}+\int \boldsymbol{v}_{d} \cdot \nabla f d^{3} \boldsymbol{v}=0
$$

Because $\int \boldsymbol{v}_{d} \cdot \nabla f d^{3} \boldsymbol{v}$ is the smallest order term appearing in the equation, we can use the lowest order solution of $f$ here. The lowest order solution for $f$ from the GKE is:

$$
f=\frac{e}{T} \frac{\omega-\omega_{*}}{\omega-k_{\|} U} F_{0}\left(\phi-\frac{k_{\|} U}{\omega} \psi_{\|}\right)+\frac{\partial F_{0}}{\partial \varepsilon} H_{1} .
$$

Substituting this solution into the quasineutrality condition, ignoring all the FLR effects, and making use of the usual cold-ion and hot-electron expansions, we easily get the expected relationship between $\psi_{\|}$and $\phi$,

$$
\psi_{\|}=\phi
$$

This is consistent with ideal MHD in which

$$
\boldsymbol{E}=\frac{1}{c} \boldsymbol{v} \times \boldsymbol{B}
$$


It is obvious that

$$
\boldsymbol{E}_{\|}=-\nabla_{\|} \phi-\frac{1}{c} \frac{\partial \boldsymbol{A}_{\|}}{\partial t}=-\nabla_{\|}\left(\phi-\psi_{\|}\right)=0
$$

Therefore $\phi=\psi_{\|}$in the ideal MHD limit.

When the solution for $f$ and the relationship between $\phi$ and $\psi_{\|}$are substituted into Equation(89), the eigenequation is formed:

$$
\frac{1}{R_{0}^{2}}\left[\omega^{2} L_{1}(\phi)+L_{2}(\phi)\right]+\frac{1}{R_{0}^{4}}\left[S^{s}(\phi)-\frac{R_{0}^{2} 8 \pi p_{0}^{\prime}(r) q^{\prime}(r)}{B_{0}^{2} q^{3}(r)}\right]=0
$$

where

$$
\begin{gathered}
L_{1}(\phi)=-\frac{1}{\omega_{A 0}^{2}}\left[\frac{1}{r} \frac{d}{d r}\left(r \rho \frac{d \phi}{d r}\right)-\rho \frac{m^{2} \phi}{r^{2}}\right], \\
L_{2}(\phi)=R_{0}^{2}\left[\frac{1}{r} \frac{d}{d r}\left(r k_{\| 0} \frac{d \phi}{d r}\right)-k_{\| 0}^{2} \frac{m^{2} \phi}{r^{2}}-\frac{1}{r} \frac{d k_{\| 0}^{2}}{d r} \phi\right],
\end{gathered}
$$

and

$$
k_{\| 0} \equiv \frac{1}{R_{0}}\left(n-\frac{m}{q}\right)
$$

Performing the operation $\int_{0}^{a} d r r \phi$ on Equation (94), we get

$$
\frac{\omega^{2}}{\omega_{A 0}^{2}}=\frac{\delta W}{\int_{0}^{a}\left[r \rho\left(\frac{d \phi}{d r}\right)^{2}+\rho \frac{m^{2} \phi^{2}}{r}\right] d r}
$$


where

$$
\delta W=\delta W_{2}+\delta W_{4}
$$

$\delta W_{2}$ is the 2 nd order contribution

$$
\delta W_{2}=R_{0}^{2} \int_{0}^{a} \frac{k_{\| 0}^{2}}{r}\left[\left(\phi-r \frac{d \phi}{d r}\right)^{2}+\left(m^{2}-1\right) \phi^{2}\right] d r
$$

and $\delta W_{4}$ is the 4 th order contribution ,

$$
\delta W_{4}=\frac{-1}{R_{0}^{2}} \int_{0}^{a} r \phi\left\{S_{\psi}^{s}[\phi]-\frac{R_{0}^{2} 8 \pi p_{0}^{\prime}(r) q^{\prime}(r)}{B_{0}^{2} q^{3}(r)}\right\} d r
$$

Using the fact that $L_{1}$ and $L_{2}$ are Sturm-Liouville operators, we can show that if for all trail functions $\delta W>0$, then all modes are stable; if there exits a trial function $\phi$ for which $\delta W<0$, then there is at least one unstable eigenmode.

We immediately reach the following conclusions:

- If $m^{2} \neq 1$, then the modes are stable with $\delta W \sim O\left(\epsilon^{2}\right)$.

- If $m^{2}=1, q(r=0)>1 / n$, then the modes are stable with $\delta W \sim O\left(\epsilon^{2}\right)$.

For the case of $m^{2}=1$ and $q(r=0)<1 / n(\operatorname{assuming} q(r=a)>1 / n)$, there exists a rational surface at $r_{s}$. We can choose the trail function as

$$
\phi(r)= \begin{cases}r, & r<r_{s} \\ 0, & r>r_{s} .\end{cases}
$$


It is obvious that $\delta W_{2}=0$ and

$$
\delta W=\delta W_{4} \sim O\left(\epsilon^{4}\right)
$$

The mode is neutral to the order $O\left(\epsilon^{2}\right)$, and the instability is determined by $\delta W_{4}$. Using the familiar family of $q$ profiles:

$$
q=q_{0} \frac{(1+\nu) r^{2}}{1-\left(1-r^{2}\right)^{1+\nu}}
$$

we can verify that for a wide range of $\nu$ and $q_{0}, \delta W_{4}$ is indeed less than zero. Therefore the $m=1$ internal kink mode is unstable when $q(r=0)<1 / n$. In Figure 4 we plot $\delta W_{4}$ against $q_{0}$ and $\nu$ for the $(n, m)=(1,1)$ case.

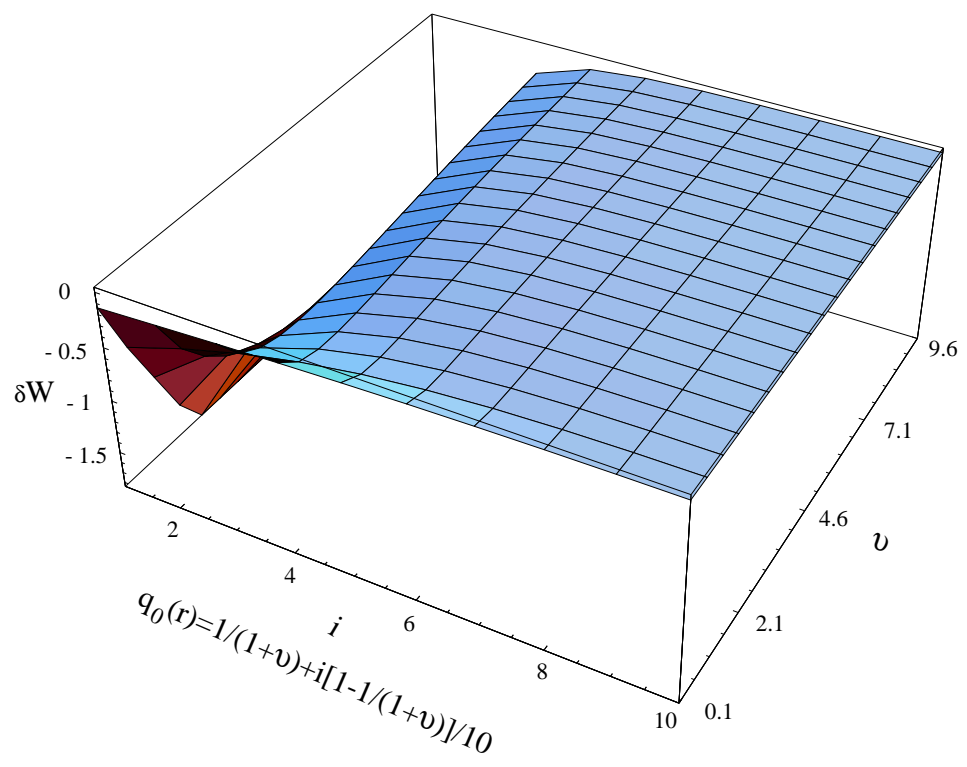

Figure 4: The gyrokinetic result for $\delta W$ for a straight tokamak.

To compare with the ideal MHD result, we also plot the minimizing $\delta W$ from the ideal MHD energy principle for the same case[16](see Figure 5). Our kinetic results agree with 


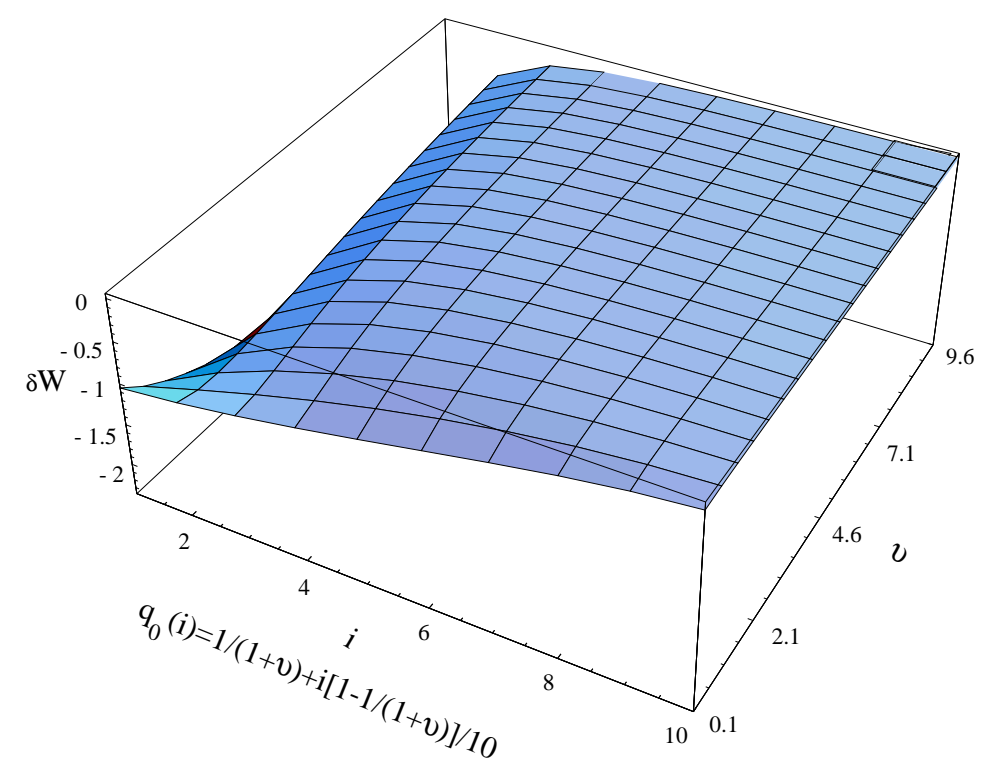

Figure 5: The ideal MHD result for $\delta W$ for a straight tokamak.

the classical ideal MHD results both quantitatively and qualitatively.

\section{Conclusions}

The gyrokinetic system for arbitrary wavelength electromagnetic modes developed in this paper can cover a wide range of phenomena in inhomogeneous plasmas, from the electrostatic drift waves to ideal MHD modes, from the short wavelength ballooning modes to the long wavelength kink modes. Even though this system is comprehensive, it is also extremely accurate. As we have seen, this system is capable of recovering the delicate internal kink mode which can't be recovered by all the existing reduced systems such as Strauss's reduced MHD. With newly developed symbolic computation facilities and the 2D comprehensive numerical code, our fully kinetic approach enables us to investigate important kinetic-MHD modes selfconsistently in great detail. It is an effective equation system to use to study the 
multi-scale-length behavior as well. These topic will be the focus of our future work and publications.

\section{Appendix A Expressions for terms in the GKM in cir- cular concentric tokamak geometry}

$$
\begin{aligned}
& O_{2}=\left(\frac{-2 m n}{q(r)}+\frac{m^{2}}{q(r)^{2}}+n^{2}\right) \psi_{\|}^{\prime \prime}(r)-\frac{4 \omega^{2} R_{0}^{2} \pi \rho(r)}{B_{0}{ }^{2}} \phi^{\prime \prime}(r)+\left(\frac{2 m n q^{\prime}(r)}{q(r)^{2}}-\frac{2 m^{2} q^{\prime}(r)}{q(r)^{3}}\right. \\
& \left.-\frac{2 m n}{r q(r)}+\frac{m^{2}}{r q(r)^{2}}+\frac{n^{2}}{r}\right) \psi_{\|}^{\prime}(r)+\left(\frac{-4 \omega^{2} R_{0}^{2} \pi \rho^{\prime}(r)}{B_{0}{ }^{2}}-\frac{4 \omega^{2} R_{0}^{2} \pi \rho(r)}{B_{0}{ }^{2} r}\right) \phi^{\prime}(r) \\
& +\left(\frac{-2 m n q^{\prime}(r)}{r q(r)^{2}}+\frac{2 m^{2} q^{\prime}(r)}{r q(r)^{3}}+\frac{2 m^{3} n}{r^{2} q(r)}-\frac{m^{4}}{r^{2} q(r)^{2}}-\frac{m^{2} n^{2}}{r^{2}}\right) \psi_{\|}(r)+\frac{4 m^{2} \omega^{2} R_{0}^{2} \pi \rho(r)}{B_{0}{ }^{2} r^{2}} \phi(r) . \\
& O_{3}=\left(\frac{-i m r \sin (\theta)}{q(r)^{2}}+\frac{2 m n r \cos (\theta)}{q(r)}-2 n^{2} r \cos (\theta)\right) \psi_{\|}^{\prime \prime}(r)-\frac{8 \omega^{2} R_{0}^{2} \pi r \cos (\theta) \rho(r)}{B_{0}{ }^{2}} \phi^{\prime \prime}(r) \\
& +\left(\frac{2 i m r \sin (\theta) q^{\prime}(r)}{q(r)^{3}}-\frac{2 m n r \cos (\theta) q^{\prime}(r)}{q(r)^{2}}-\frac{2 i n \sin (\theta)}{q(r)}-\frac{i m \sin (\theta)}{q(r)^{2}}+\frac{2 m n \cos (\theta)}{q(r)}\right. \\
& \left.+\frac{m^{2} \cos (\theta)}{q(r)^{2}}-3 n^{2} \cos (\theta)\right) \psi_{\|}^{\prime}(r)+\left(\frac{-8 \omega^{2} R_{0}^{2} \pi r \cos (\theta) \rho^{\prime}(r)}{B_{0}{ }^{2}}-\frac{20 \omega^{2} R_{0}^{2} \pi \cos (\theta) \rho(r)}{B_{0}{ }^{2}}\right) \phi^{\prime}(r) \\
& +\left(\frac{-2 i m \sin (\theta) q^{\prime}(r)}{q(r)^{3}}+\frac{4 m n \cos (\theta) q^{\prime}(r)}{q(r)^{2}}-\frac{2 m^{2} \cos (\theta) q^{\prime}(r)}{q(r)^{3}}+\frac{2 i m^{3} \sin (\theta)}{r q(r)^{2}}\right. \\
& \left.-\frac{i m n^{2} \sin (\theta)}{r}-\frac{2 m^{3} n \cos (\theta)}{r q(r)}-\frac{2 m n \cos (\theta)}{r q(r)}+\frac{2 m^{2} n^{2} \cos (\theta)}{r}\right) \psi_{\|}(r) \\
& +\left(\frac{-12 i m \omega^{2} R_{0}^{2} \pi \rho(r) \sin (\theta)}{B_{0}{ }^{2} r}+\frac{8 m^{2} \omega^{2} R_{0}^{2} \pi \cos (\theta) \rho(r)}{B_{0}{ }^{2} r}\right) \phi(r) .
\end{aligned}
$$




$$
\begin{aligned}
D_{1 \psi} & =\left(-\left(n^{2} r\right)-\frac{m r}{2 q(r)^{2}}+\frac{m n r}{q(r)}\right) \psi_{\|}^{\prime \prime}(r)+\left(\frac{-3 n^{2}}{2}-\frac{m}{2 q(r)^{2}}+\frac{m^{2}}{2 q(r)^{2}}-\frac{n}{q(r)}+\frac{m n}{q(r)}\right. \\
& \left.+\frac{m r q^{\prime}(r)}{q(r)^{3}}-\frac{m n r q^{\prime}(r)}{q(r)^{2}}\right) \psi_{\|}^{\prime}(r)+\left(\frac{-\left(m n^{2}\right)}{2 r}+\frac{m^{2} n^{2}}{r}+\frac{m^{3}}{r q(r)^{2}}\right. \\
& \left.-\frac{m n}{r q(r)}-\frac{m^{3} n}{r q(r)}-\frac{m q^{\prime}(r)}{q(r)^{3}}-\frac{m^{2} q^{\prime}(r)}{q(r)^{3}}+\frac{2 m n q^{\prime}(r)}{q(r)^{2}}\right) \psi_{\|}(r) \\
D_{1 \phi} & =\frac{-4 \omega^{2} R_{0}^{2} \pi r \rho(r)}{B_{0}^{2}} \phi^{\prime \prime}(r)+\left(\frac{-10 \omega^{2} R_{0}^{2} \pi \rho(r)}{B_{0}^{2}}-\frac{4 \omega^{2} R_{0}^{2} \pi r \rho^{\prime}(r)}{B_{0}{ }^{2}}\right) \phi^{\prime}(r) \\
& +\left(\frac{-6 m \omega^{2} R_{0}^{2} \pi \rho(r)}{B_{0}{ }^{2} r}+\frac{4 m^{2} \omega^{2} R_{0}^{2} \pi \rho(r)}{B_{0}{ }^{2} r}\right) \phi(r) .
\end{aligned}
$$

$$
\begin{aligned}
& U_{1 \psi}=\left(-\left(n^{2} r\right)+\frac{m r}{2 q(r)^{2}}+\frac{m n r}{q(r)}\right) \psi_{\|}^{\prime \prime}(r)+\left(\frac{-3 n^{2}}{2}+\frac{m}{2 q(r)^{2}}+\frac{m^{2}}{2 q(r)^{2}}+\frac{n}{q(r)}+\frac{m n}{q(r)}\right. \\
& \left.-\frac{m r q^{\prime}(r)}{q(r)^{3}}-\frac{m n r q^{\prime}(r)}{q(r)^{2}}\right) \psi_{\|}^{\prime}(r)+\left(\frac{m n^{2}}{2 r}+\frac{m^{2} n^{2}}{r}-\frac{m^{3}}{r q(r)^{2}}\right. \\
& \left.-\frac{m n}{r q(r)}-\frac{m^{3} n}{r q(r)}+\frac{m q^{\prime}(r)}{q(r)^{3}}-\frac{m^{2} q^{\prime}(r)}{q(r)^{3}}+\frac{2 m n q^{\prime}(r)}{q(r)^{2}}\right) \psi_{\|}(r) . \\
& U_{1 \phi}=\frac{-4 \omega^{2} R_{0}^{2} \pi r \rho(r)}{B_{0}{ }^{2}} \phi^{\prime \prime}(r)+\left(\frac{-10 \omega^{2} R_{0}^{2} \pi \rho(r)}{B_{0}{ }^{2}}-\frac{4 \omega^{2} R_{0}^{2} \pi r \rho^{\prime}(r)}{B_{0}{ }^{2}}\right) \phi^{\prime}(r) \\
& +\left(\frac{6 m \omega^{2} R_{0}^{2} \pi \rho(r)}{B_{0}{ }^{2} r}+\frac{4 m^{2} \omega^{2} R_{0}^{2} \pi \rho(r)}{B_{0}{ }^{2} r}\right) \phi(r) .
\end{aligned}
$$




$$
\begin{aligned}
O_{4}^{t} & =\left(\frac{i m r^{2} \cos (\theta) \sin (\theta)}{q(r)^{2}}-\frac{2 m n r^{2} \cos (\theta)^{2}}{q(r)}+3 n^{2} r^{2} \cos (\theta)^{2}\right) \psi_{\|}^{\prime \prime}(r) \\
& -\frac{4 \omega^{2} R_{0}^{2} \pi r^{2} \cos (\theta)^{2} \rho(r)}{B_{0}^{2}} \phi^{\prime \prime}(r)+\left(\frac{-2 i m r^{2} \cos (\theta) \sin (\theta) q^{\prime}(r)}{q(r)^{3}}+\frac{2 m n r^{2} \cos (\theta)^{2} q^{\prime}(r)}{q(r)^{2}}\right. \\
& \left.+\frac{6 i n r \cos (\theta) \sin (\theta)}{q(r)}-\frac{2 m n r \cos (\theta)^{2}}{q(r)}-\frac{m^{2} r \cos (\theta)^{2}}{q(r)^{2}}+6 n^{2} r \cos (\theta)^{2}\right) \psi_{\|}^{\prime}(r) \\
& +\left(\frac{-4 \omega^{2} R_{0}^{2} \pi r^{2} \cos (\theta)^{2} \rho^{\prime}(r)}{B_{0}{ }^{2}}--\frac{16 \omega^{2} R_{0}^{2} \pi r \cos (\theta)^{2} \rho(r)}{B_{0}{ }^{2}}\right) \phi^{\prime}(r) \\
& +\left(\frac{4 i m r \cos (\theta) \sin (\theta) q^{\prime}(r)}{q(r)^{3}}-\frac{6 m n r \cos (\theta)^{2} q^{\prime}(r)}{q(r)^{2}}+\frac{2 m^{2} r \cos (\theta)^{2} q^{\prime}(r)}{q(r)^{3}}-\frac{m^{2} \sin (\theta)^{2}}{q(r)^{2}}\right. \\
& -\frac{2 i m^{3} \cos (\theta) \sin (\theta)}{q(r)^{2}}+3 i m n^{2} \cos (\theta) \sin (\theta)+\frac{2 m^{3} n \cos (\theta)^{2}}{q(r)}+\frac{6 m n \cos (\theta)^{2}}{q(r)} \\
& \left.-\frac{2 m^{2} \cos (\theta)^{2}}{q(r)^{2}}-3 m^{2} n^{2} \cos (\theta)^{2}\right) \psi_{\|}(r) \\
& +\left(\frac{-12 i m \omega^{2} R_{0}^{2} \pi \cos (\theta) \rho(r) \sin (\theta)}{B_{0}{ }^{2}}+\frac{4 m^{2} \omega^{2} R_{0}^{2} \pi \cos (\theta)^{2} \rho(r)}{B_{0}{ }^{2}}\right) \phi(r) .
\end{aligned}
$$

$$
\begin{aligned}
O_{4}^{s} & =\left(-\frac{n^{2} r^{2}}{q(r)^{2}}+\frac{2 m n r^{2}}{q(r)^{3}}-\frac{m^{2} r^{2}}{q(r)^{4}}\right) \psi_{\|}^{\prime \prime}(r)+\frac{4 \omega^{2} R_{0}^{2} \pi r^{2} \rho(r)}{B_{0}^{2} q(r)^{2}} \phi^{\prime \prime}(r)+\left(\frac{2 n^{2} r^{2} q^{\prime}(r)}{q(r)^{3}}\right. \\
& \left.-\frac{6 m n r^{2} q^{\prime}(r)}{q(r)^{4}}+\frac{4 m^{2} r^{2} q^{\prime}(r)}{q(r)^{5}}-\frac{3 n^{2} r}{q(r)^{2}}+\frac{6 m n r}{q(r)^{3}}-\frac{3 m^{2} r}{q(r)^{4}}\right) \psi_{\|}^{\prime}(r)+\left(\frac{4 \omega^{2} R_{0}^{2} \pi r^{2} \rho^{\prime}(r)}{B_{0}^{2} q(r)^{2}}\right. \\
& \left.-\frac{8 \omega^{2} R_{0}^{2} \pi r^{2} \rho(r) q^{\prime}(r)}{B_{0}^{2} q(r)^{3}}+\frac{12 \omega^{2} R_{0}^{2} \pi r \rho(r)}{B_{0}^{2} q(r)^{2}}\right) \phi^{\prime}(r)+\left(-\frac{n^{2} r^{2} q^{\prime}(r)^{2}}{q(r)^{4}}+\frac{2 m n r^{2} q^{\prime}(r)^{2}}{q(r)^{5}}\right. \\
& -\frac{m^{2} r^{2} q^{\prime}(r)^{2}}{q(r)^{6}}+\frac{6 m n r q^{\prime}(r)}{q(r)^{4}}-\frac{6 m^{2} r q^{\prime}(r)}{q(r)^{5}}-\frac{2 m n^{3}}{q(r)}+\frac{6 m^{2} n^{2}}{q(r)^{2}}-\frac{6 m^{3} n}{q(r)^{3}}-\frac{4 m n}{q(r)^{3}} \\
& \left.+\frac{2 m^{4}}{q(r)^{4}}+\frac{4 m^{2}}{q(r)^{4}}\right) \psi_{\|}(r)+\left(\frac{8 m n \omega^{2} R_{0}^{2} \pi \rho(r)}{B_{0}^{2} q(r)}-\frac{8 m^{2} \omega^{2} R_{0}^{2} \pi \rho(r)}{B_{0}{ }^{2} q(r)^{2}}\right) \phi(r) .
\end{aligned}
$$




$$
\begin{aligned}
& O_{4}=\left(\frac{\frac{i}{2} m r^{2} \sin (2 \theta)}{q(r)^{2}}-\frac{m n r^{2} \cos (2 \theta)}{q(r)}-\frac{m n r^{2}}{q(r)}-\frac{n^{2} r^{2}}{q(r)^{2}}+\frac{2 m n r^{2}}{q(r)^{3}}-\frac{m^{2} r^{2}}{q(r)^{4}}\right. \\
& \left.+\frac{3 n^{2} r^{2} \cos (2 \theta)}{2}+\frac{3 n^{2} r^{2}}{2}\right) \psi_{\|}^{\prime \prime}(r)+\left(\frac{4 \omega^{2} R_{0}^{2} \pi r^{2} \rho(r)}{B_{0}{ }^{2} q(r)^{2}}-\frac{2 \omega^{2} R_{0}^{2} \pi r^{2} \cos (2 \theta) \rho(r)}{B_{0}{ }^{2}}\right. \\
& \left.-\frac{2 \omega^{2} R_{0}^{2} \pi r^{2} \rho(r)}{B_{0}{ }^{2}}\right) \phi^{\prime \prime}(r)+\left(\frac{-i m r^{2} \sin (2 \theta) q^{\prime}(r)}{q(r)^{3}}+\frac{m n r^{2} \cos (2 \theta) q^{\prime}(r)}{q(r)^{2}}+\frac{m n r^{2} q^{\prime}(r)}{q(r)^{2}}\right. \\
& +\frac{2 n^{2} r^{2} q^{\prime}(r)}{q(r)^{3}}-\frac{6 m n r^{2} q^{\prime}(r)}{q(r)^{4}}+\frac{4 m^{2} r^{2} q^{\prime}(r)}{q(r)^{5}}+\frac{3 i n r \sin (2 \theta)}{q(r)}-\frac{m n r \cos (2 \theta)}{q(r)}-\frac{m n r}{q(r)} \\
& \left.-\frac{m^{2} r \cos (2 \theta)}{2 q(r)^{2}}-\frac{3 n^{2} r}{q(r)^{2}}-\frac{m^{2} r}{2 q(r)^{2}}+\frac{6 m n r}{q(r)^{3}}-\frac{3 m^{2} r}{q(r)^{4}}+3 n^{2} r \cos (2 \theta)+3 n^{2} r\right) \psi_{\|}^{\prime}(r) \\
& +\left(\frac{4 \omega^{2} R_{0}^{2} \pi r^{2} \rho^{\prime}(r)}{B_{0}{ }^{2} q(r)^{2}}-\frac{2 \omega^{2} R_{0}^{2} \pi r^{2} \cos (2 \theta) \rho^{\prime}(r)}{B_{0}{ }^{2}}-\frac{2 \omega^{2} R_{0}^{2} \pi r^{2} \rho^{\prime}(r)}{B_{0}{ }^{2}}-\frac{8 \omega^{2} R_{0}^{2} \pi r^{2} \rho(r) q^{\prime}(r)}{B_{0}{ }^{2} q(r)^{3}}\right. \\
& \left.+\frac{12 \omega^{2} R_{0}^{2} \pi r \rho(r)}{B_{0}{ }^{2} q(r)^{2}}-\frac{8 \omega^{2} R_{0}^{2} \pi r \cos (2 \theta) \rho(r)}{B_{0}{ }^{2}}-\frac{8 \omega^{2} R_{0}^{2} \pi r \rho(r)}{B_{0}{ }^{2}}\right) \phi^{\prime}(r)+\left(-\frac{n^{2} r^{2} q^{\prime}(r)^{2}}{q(r)^{4}}\right. \\
& +\frac{2 m n r^{2} q^{\prime}(r)^{2}}{q(r)^{5}}-\frac{m^{2} r^{2} q^{\prime}(r)^{2}}{q(r)^{6}}+\frac{2 i m r \sin (2 \theta) q^{\prime}(r)}{q(r)^{3}}-\frac{3 m n r \cos (2 \theta) q^{\prime}(r)}{q(r)^{2}} \\
& -\frac{3 m n r q^{\prime}(r)}{q(r)^{2}}+\frac{m^{2} r \cos (2 \theta) q^{\prime}(r)}{q(r)^{3}}+\frac{m^{2} r q^{\prime}(r)}{q(r)^{3}}+\frac{6 m n r q^{\prime}(r)}{q(r)^{4}}-\frac{6 m^{2} r q^{\prime}(r)}{q(r)^{5}} \\
& -\frac{i m^{3} \sin (2 \theta)}{q(r)^{2}}+\frac{3 i}{2} m n^{2} \sin (2 \theta)+\frac{m^{3} n \cos (2 \theta)}{q(r)}+\frac{3 m n \cos (2 \theta)}{q(r)}-\frac{2 m n^{3}}{q(r)} \\
& +\frac{m^{3} n}{q(r)}+\frac{3 m n}{q(r)}-\frac{m^{2} \cos (2 \theta)}{2 q(r)^{2}}+\frac{6 m^{2} n^{2}}{q(r)^{2}}-\frac{3 m^{2}}{2 q(r)^{2}}-\frac{6 m^{3} n}{q(r)^{3}}-\frac{4 m n}{q(r)^{3}}+\frac{2 m^{4}}{q(r)^{4}}+\frac{4 m^{2}}{q(r)^{4}} \\
& \left.-\frac{3 m^{2} n^{2} \cos (2 \theta)}{2}-\frac{3 m^{2} n^{2}}{2}\right) \psi_{\|}(r)+\left(\frac{-6 i m \omega^{2} R_{0}^{2} \pi \rho(r) \sin (2 \theta)}{B_{0}{ }^{2}}+\frac{8 m n \omega^{2} R_{0}^{2} \pi \rho(r)}{B_{0}{ }^{2} q(r)}\right. \\
& \left.-\frac{8 m^{2} \omega^{2} R_{0}^{2} \pi \rho(r)}{B_{0}{ }^{2} q(r)^{2}}+\frac{2 m^{2} \omega^{2} R_{0}^{2} \pi \cos (2 \theta) \rho(r)}{B_{0}{ }^{2}}+\frac{2 m^{2} \omega^{2} R_{0}^{2} \pi \rho(r)}{B_{0}{ }^{2}}\right) \phi(r) .
\end{aligned}
$$




$$
\begin{aligned}
D_{2 \psi} & =\left(\frac{3 n^{2} r^{2}}{4}+\frac{m r^{2}}{4 q(r)^{2}}-\frac{m n r^{2}}{2 q(r)}\right) \psi_{\|}^{\prime \prime}(r)+\left(\frac{3 n^{2} r}{2}-\frac{m^{2} r}{4 q(r)^{2}}+\frac{3 n r}{2 q(r)}\right. \\
& \left.-\frac{m n r}{2 q(r)}-\frac{m r^{2} q^{\prime}(r)}{2 q(r)^{3}}+\frac{m n r^{2} q^{\prime}(r)}{2 q(r)^{2}}\right) \psi_{\|}^{\prime}(r)+\left(\frac{3 m n^{2}}{4}-\frac{3 m^{2} n^{2}}{4}-\frac{m^{2}}{4 q(r)^{2}}-\frac{m^{3}}{2 q(r)^{2}}\right. \\
& \left.+\frac{3 m n}{2 q(r)}+\frac{m^{3} n}{2 q(r)}+\frac{m r q^{\prime}(r)}{q(r)^{3}}+\frac{m^{2} r q^{\prime}(r)}{2 q(r)^{3}}-\frac{3 m n r q^{\prime}(r)}{2 q(r)^{2}}\right) \psi_{\|}(r) .
\end{aligned}
$$

$$
\begin{aligned}
D_{2 \phi} & =-\frac{\omega^{2} R_{0}^{2} \pi r^{2} \rho(r) \phi^{\prime \prime}(r)}{B_{0}{ }^{2}}+\left(\frac{-4 \omega^{2} R_{0}^{2} \pi r \rho(r)}{B_{0}{ }^{2}}-\frac{\omega^{2} R_{0}^{2} \pi r^{2} \rho^{\prime}(r)}{B_{0}{ }^{2}}\right) \phi^{\prime}(r)+\left(\frac{-3 m \omega^{2} R_{0}^{2} \pi \rho(r)}{B_{0}{ }^{2}}\right. \\
& \left.+\frac{m^{2} \omega^{2} R_{0}^{2} \pi \rho(r)}{B_{0}{ }^{2}}\right) \phi(r) .
\end{aligned}
$$

$$
\begin{aligned}
U_{2 \psi} & =\left(\frac{3 n^{2} r^{2}}{4}-\frac{m r^{2}}{4 q(r)^{2}}-\frac{m n r^{2}}{2 q(r)}\right) \psi_{\|}^{\prime \prime}(r)+\left(\frac{3 n^{2} r}{2}-\frac{m^{2} r}{4 q(r)^{2}}-\frac{3 n r}{2 q(r)}-\frac{m n r}{2 q(r)}+\frac{m r^{2} q^{\prime}(r)}{2 q(r)^{3}}\right. \\
& \left.+\frac{m n r^{2} q^{\prime}(r)}{2 q(r)^{2}}\right) \psi_{\|}^{\prime}(r)+\left(\frac{-3 m n^{2}}{4}-\frac{3 m^{2} n^{2}}{4}-\frac{m^{2}}{4 q(r)^{2}}+\frac{m^{3}}{2 q(r)^{2}}+\frac{3 m n}{2 q(r)}+\frac{m^{3} n}{2 q(r)}\right. \\
& \left.-\frac{m r q^{\prime}(r)}{q(r)^{3}}+\frac{m^{2} r q^{\prime}(r)}{2 q(r)^{3}}-\frac{3 m n r q^{\prime}(r)}{2 q(r)^{2}}\right) \psi_{\|}(r) .
\end{aligned}
$$

$$
\begin{aligned}
U_{2 \phi} & =-\frac{\omega^{2} R_{0}^{2} \pi r^{2} \rho(r) \phi^{\prime \prime}(r)}{B_{0}{ }^{2}}+\left(\frac{-4 \omega^{2} R_{0}^{2} \pi r \rho(r)}{B_{0}{ }^{2}}-\frac{\omega^{2} R_{0}^{2} \pi r^{2} \rho^{\prime}(r)}{B_{0}{ }^{2}}\right) \phi^{\prime}(r)+\left(\frac{3 m \omega^{2} R_{0}^{2} \pi \rho(r)}{B_{0}{ }^{2}}\right. \\
& \left.+\frac{m^{2} \omega^{2} R_{0}^{2} \pi \rho(r)}{B_{0}{ }^{2}}\right) \phi(r) .
\end{aligned}
$$




$$
\begin{aligned}
S_{\psi} & =\left(\frac{3 n^{2} r^{2}}{2}-\frac{m^{2} r^{2}}{q(r)^{4}}+\frac{2 m n r^{2}}{q(r)^{3}}-\frac{n^{2} r^{2}}{q(r)^{2}}-\frac{m n r^{2}}{q(r)}\right) \psi_{\|}^{\prime \prime}(r)+\left(3 n^{2} r-\frac{3 m^{2} r}{q(r)^{4}}+\frac{6 m n r}{q(r)^{3}}\right. \\
& -\frac{m^{2} r}{2 q(r)^{2}}-\frac{3 n^{2} r}{q(r)^{2}}-\frac{m n r}{q(r)}+\frac{4 m^{2} r^{2} q^{\prime}(r)}{q(r)^{5}}-\frac{6 m n r^{2} q^{\prime}(r)}{q(r)^{4}}+\frac{2 n^{2} r^{2} q^{\prime}(r)}{q(r)^{3}} \\
& \left.+\frac{m n r^{2} q^{\prime}(r)}{q(r)^{2}}\right) \psi_{\|}^{\prime}(r)+\left(\frac{-3 m^{2} n^{2}}{2}+\frac{4 m^{2}}{q(r)^{4}}+\frac{2 m^{4}}{q(r)^{4}}-\frac{4 m n}{q(r)^{3}}-\frac{6 m^{3} n}{q(r)^{3}}-\frac{3 m^{2}}{2 q(r)^{2}}\right. \\
& +\frac{6 m^{2} n^{2}}{q(r)^{2}}+\frac{3 m n}{q(r)}+\frac{m^{3} n}{q(r)}-\frac{2 m n^{3}}{q(r)}-\frac{6 m^{2} r q^{\prime}(r)}{q(r)^{5}}+\frac{6 m n r q^{\prime}(r)}{q(r)^{4}}+\frac{m^{2} r q^{\prime}(r)}{q(r)^{3}} \\
& \left.-\frac{3 m n r q^{\prime}(r)}{q(r)^{2}}-\frac{m^{2} r^{2} q^{\prime}(r)^{2}}{q(r)^{6}}+\frac{2 m n r^{2} q^{\prime}(r)^{2}}{q(r)^{5}}-\frac{n^{2} r^{2} q^{\prime}(r)^{2}}{q(r)^{4}}\right) \psi_{\|}(r) .
\end{aligned}
$$

$$
\begin{aligned}
S_{\phi} & =\left(\frac{-2 \omega^{2} R_{0}^{2} \pi r^{2} \rho(r)}{B_{0}{ }^{2}}+\frac{4 \omega^{2} R_{0}^{2} \pi r^{2} \rho(r)}{B_{0}{ }^{2} q(r)^{2}}\right) \phi^{\prime \prime}(r)+\left(\frac{-8 \omega^{2} R_{0}^{2} \pi r \rho(r)}{B_{0}{ }^{2}}\right. \\
& \left.+\frac{12 \omega^{2} R_{0}^{2} \pi r \rho(r)}{B_{0}{ }^{2} q(r)^{2}}-\frac{8 \omega^{2} R_{0}^{2} \pi r^{2} \rho(r) q^{\prime}(r)}{B_{0}{ }^{2} q(r)^{3}}-\frac{2 \omega^{2} R_{0}^{2} \pi r^{2} \rho^{\prime}(r)}{B_{0}{ }^{2}}+\frac{4 \omega^{2} R_{0}^{2} \pi r^{2} \rho^{\prime}(r)}{B_{0}{ }^{2} q(r)^{2}}\right) \phi^{\prime}(r) \\
& +\left(\frac{2 m^{2} \omega^{2} R_{0}^{2} \pi \rho(r)}{B_{0}{ }^{2}}-\frac{8 m^{2} \omega^{2} R_{0}^{2} \pi \rho(r)}{B_{0}{ }^{2} q(r)^{2}}+\frac{8 m n \omega^{2} R_{0}^{2} \pi \rho(r)}{B_{0}{ }^{2} q(r)}\right) \phi(r) .
\end{aligned}
$$

$$
\begin{aligned}
S_{\psi}^{s} & =\left(-\frac{m^{2} r^{2}}{q(r)^{4}}+\frac{2 m n r^{2}}{q(r)^{3}}-\frac{n^{2} r^{2}}{q(r)^{2}}\right) \psi_{\|}^{\prime \prime}(r)+\left(\frac{-3 m^{2} r}{q(r)^{4}}+\frac{6 m n r}{q(r)^{3}}-\frac{3 n^{2} r}{q(r)^{2}}\right. \\
& \left.+\frac{4 m^{2} r^{2} q^{\prime}(r)}{q(r)^{5}}-\frac{6 m n r^{2} q^{\prime}(r)}{q(r)^{4}}+\frac{2 n^{2} r^{2} q^{\prime}(r)}{q(r)^{3}}\right) \psi_{\|}^{\prime}(r) \\
& +\left(\frac{4 m^{2}}{q(r)^{4}}+\frac{2 m^{4}}{q(r)^{4}}-\frac{4 m n}{q(r)^{3}}-\frac{6 m^{3} n}{q(r)^{3}}+\frac{6 m^{2} n^{2}}{q(r)^{2}}-\frac{2 m n^{3}}{q(r)}\right. \\
& \left.-\frac{6 m^{2} r q^{\prime}(r)}{q(r)^{5}}+\frac{6 m n r q^{\prime}(r)}{q(r)^{4}}-\frac{m^{2} r^{2} q^{\prime}(r)^{2}}{q(r)^{6}}+\frac{2 m n r^{2} q^{\prime}(r)^{2}}{q(r)^{5}}-\frac{n^{2} r^{2} q^{\prime}(r)^{2}}{q(r)^{4}}\right) \psi_{\|}(r) .
\end{aligned}
$$




$$
\begin{aligned}
S_{\phi}^{s} & =\frac{4 \omega^{2} R_{0}^{2} \pi r^{2} \rho(r) \phi^{\prime \prime}(r)}{B_{0}^{2} q(r)^{2}}+\left(\frac{12 \omega^{2} R_{0}^{2} \pi r \rho(r)}{B_{0}^{2} q(r)^{2}}-\frac{8 \omega^{2} R_{0}^{2} \pi r^{2} \rho(r) q^{\prime}(r)}{B_{0}{ }^{2} q(r)^{3}}\right. \\
& \left.+\frac{4 \omega^{2} R_{0}^{2} \pi r^{2} \rho^{\prime}(r)}{B_{0}^{2} q(r)^{2}}\right) \phi^{\prime}(r)+\left(\frac{-8 m^{2} \omega^{2} R_{0}^{2} \pi \rho(r)}{B_{0}^{2} q(r)^{2}}+\frac{8 m n \omega^{2} R_{0}^{2} \pi \rho(r)}{B_{0}^{2} q(r)}\right) \phi(r) . \\
S_{\psi}^{t} & =\left(\frac{3 n^{2} r^{2}}{2}-\frac{m n r^{2}}{q(r)}\right) \psi_{\|}^{\prime \prime}(r)+\left(3 n^{2} r-\frac{m^{2} r}{2 q(r)^{2}}-\frac{m n r}{q(r)}+\frac{m n r^{2} q^{\prime}(r)}{q(r)^{2}}\right) \psi_{\|}^{\prime}(r) \\
& +\left(\frac{-3 m^{2} n^{2}}{2}-\frac{3 m^{2}}{2 q(r)^{2}}+\frac{3 m n}{q(r)}+\frac{m^{3} n}{q(r)}+\frac{m^{2} r q^{\prime}(r)}{q(r)^{3}}-\frac{3 m n r q^{\prime}(r)}{q(r)^{2}}\right) \psi_{\|}(r) . \\
S_{\phi}^{t} & =\frac{-2 \omega^{2} R_{0}^{2} \pi r^{2} \rho(r) \phi^{\prime \prime}(r)}{B_{0}{ }^{2}}+\left(\frac{-8 \omega^{2} R_{0}^{2} \pi r \rho(r)}{B_{0}{ }^{2}}-\frac{2 \omega^{2} R_{0}^{2} \pi r^{2} \rho^{\prime}(r)}{B_{0}{ }^{2}}\right) \phi^{\prime}(r) \\
& +\frac{2 m^{2} \omega^{2} R_{0}^{2} \pi \rho(r)}{B_{0}{ }^{2}} \phi(r) .
\end{aligned}
$$

\section{References}

[1] J. Q. Dong, P. N. Guzdar, and Y. C. Lee, Phys. Fluids 30, 2694 (1987).

[2] G. Rewoldt, W. M. Tang, and M. S. Chance, Phys. Fluids 25, 480 (1982).

[3] M. Kotschenreuther, G. Rewoldt, and W. M. Tang, Comp. Phys. Comm. 88, 128 (1995).

[4] W. M. Tang, J. W. Connor, and R. J. Hastie, Nuclear Fusion 21, 1439 (1980).

[5] R. Marchand, Ph.D. Thesis, Princeton University, 1979.

[6] R. Marchand, W.M. Tang, and G. Rewoldt, Phys. Fluids 23, 1164 (1980).

[7] W. M. Tang and G. Rewoldt, Phys. Fluids B 5, 2451 (1993). 
[8] R. G. Littlejohn, Phys. Fluids 24, 1730 (1981).

[9] J. R. Carry, Phys. Rep. 79, 129 (1981).

[10] R. G. Littlejohn, J. Plasma Phys. 29, 111 (1983).

[11] T. S. Hahm, Phys. Fluids 31, 2670 (1988).

[12] A. Brizard, J. Plasma Phys. 41, 541 (1989).

[13] C. Z. Cheng and M. S. Chance, Phys. Fluids 29, 3695 (1986).

[14] G. Y. Fu and J. W. Van Dam, Phys. Fluids B 1, 1949 (1989).

[15] V. D. Shafranov, Sov. Phys.-Tech. Phys. 15, 175 (1970).

[16] M. N. Rosenbluth, R. Y. Dagazian, and P. H. Rutherford, Phys. Fluids 16, 1894 (1973).

[17] M. N. Bussac, R. Pellat, D. Edery, and J. L. Soule, Phys. Rev. Letter 35,1638 (1975).

[18] H. Qin, W. M. Tang, and G. Rewoldt, "Symbolic Vector Analysis in Plasma Physics", submitted to Computer Phys. Comm.

[19] P. J. Catto, W. M. Tang, and D. E. Baldwin, Plasma Phys. 23, 639 (1981).

[20] D. H. E. Dubin, J. A. Krommes, C. Oberman, and W. W. Lee, Phys. Fluids 26, 3524 $(1983)$.

[21] R. G. Littlejohn, Phys. Fluids 27, 976 (1984).

[22] T. S. Hahm, W. W. Lee, and A. Brizard, Phys. Fluids 31, 1940 (1988).

[23] M. N. Rosenbluth and P. H. Rutherford, Phys. Rev. Letter 34, 1428 (1975). 
[24] H. L. Berk, J. W. Van Dam, Z. Guo, and D. M. Lindberg, Phys. Fluids B 1, 1806 (1992).

[25] H. R. Strauss, Phys. Fluids 19, 134 (1976).

[26] R. D. Hazeltine and J. D. Mesis, Plasma Comfinement (Addison-Wesley, 1992), pp 267.

[27] H. Naitou, K. Tsuda, W. W. Lee and R. D. Sydora, Phys. Plasmas 2, 4257 (1995).

[28] S. Wolfram, The Mathematica Book (Wolfram Media, 1996).

[29] W. M. Tang, Nuclear Fusion 18, 1089 (1978).

[30] M. Artun, Ph.D. thesis, Princeton University, 1994.

[31] M. Artun, W. M. Tang, and G. Rewoldt, Phys. Plasmas 2, 3384 (1995). 